\title{
A matrix-less and parallel interpolation-extrapolation algorithm for computing the eigenvalues of preconditioned banded symmetric Toeplitz matrices
}

\author{
Sven-Erik Ekström ${ }^{1}$ (D) . Carlo Garoni ${ }^{2,3}$ (D)
}

Received: 22 August 2017 / Accepted: 4 March 2018 / Published online: 24 March 2018

(C) The Author(s) 2018

\begin{abstract}
In the past few years, Bogoya, Böttcher, Grudsky, and Maximenko obtained the precise asymptotic expansion for the eigenvalues of a Toeplitz matrix $T_{n}(f)$, under suitable assumptions on the generating function $f$, as the matrix size $n$ goes to infinity. On the basis of several numerical experiments, it was conjectured by Serra-Capizzano that a completely analogous expansion also holds for the eigenvalues of the preconditioned Toeplitz matrix $T_{n}(u)^{-1} T_{n}(v)$, provided $f=v / u$ is monotone and further conditions on $u$ and $v$ are satisfied. Based on this expansion, we here propose and analyze an interpolation-extrapolation algorithm for computing the eigenvalues of $T_{n}(u)^{-1} T_{n}(v)$. The algorithm is suited for parallel implementation and it may be called "matrix-less" as it does not need to store the entries of the matrix. We illustrate the performance of the algorithm through numerical experiments and we also present its generalization to the case where $f=v / u$ is non-monotone.
\end{abstract}

Keywords Preconditioned Toeplitz matrices - Eigenvalues $\cdot$ Asymptotic eigenvalue expansion $\cdot$ Polynomial interpolation $\cdot$ Extrapolation

Mathematics Subject Classification (2010) $15 \mathrm{~B} 05 \cdot 65 \mathrm{~F} 15 \cdot 65 \mathrm{D} 05 \cdot 65 \mathrm{~B} 05$

Sven-Erik Ekström

sven-erik.ekstrom@it.uu.se

Carlo Garoni

carlo.garoni@usi.ch; carlo.garoni@uninsubria.it

1 Department of Information Technology, Division of Scientific Computing, Uppsala University, ITC, Lägerhyddsv. 2, Hus 2, P.O. Box 337, SE-751 05 Uppsala, Sweden

2 Institute of Computational Science, University of Italian Switzerland (USI), Via Giuseppe Buffi 13, 6900 Lugano, Switzerland

3 Department of Science and High Technology, University of Insubria, Via Valleggio 11, 22100 Como, Italy 


\section{Introduction}

A matrix of the form

$$
\left[a_{i-j}\right]_{i, j=1}^{n}=\left[\begin{array}{ccccc}
a_{0} & a_{-1} & \cdots & \cdots & a_{-(n-1)} \\
a_{1} & \ddots & \ddots & & \vdots \\
\vdots & \ddots & \ddots & \ddots & \vdots \\
\vdots & & \ddots & \ddots & a_{-1} \\
a_{n-1} & \cdots & \cdots & a_{1} & a_{0}
\end{array}\right],
$$

whose entries are constant along each diagonal, is called a Toeplitz matrix. Given a function $g:[-\pi, \pi] \rightarrow \mathbb{C}$ belonging to $L^{1}([-\pi, \pi])$, the $n$th Toeplitz matrix associated with $g$ is defined as

$$
T_{n}(g)=\left[\hat{g}_{i-j}\right]_{i, j=1}^{n},
$$

where the numbers $\hat{g}_{k}$ are the Fourier coefficients of $g$,

$$
\hat{g}_{k}=\frac{1}{2 \pi} \int_{-\pi}^{\pi} g(\theta) \mathrm{e}^{-\mathrm{i} k \theta} \mathrm{d} \theta, \quad k \in \mathbb{Z} .
$$

We refer to $\left\{T_{n}(g)\right\}_{n}$ as the Toeplitz sequence generated by $g$, which in turn is called the generating function of $\left\{T_{n}(g)\right\}_{n}$. It is not difficult to see that, whenever $g$ is real, $T_{n}(g)$ is Hermitian for all $n$. Moreover, if $g$ is real non-negative and not almost everywhere equal to zero in $[-\pi, \pi]$, then $T_{n}(g)$ is Hermitian positive definite for all $n$; see $[9,14]$. In the case where $g$ is a real cosine trigonometric polynomial (RCTP), that is, a function of the form

$$
g(\theta)=\hat{g}_{0}+2 \sum_{k=1}^{m} \hat{g}_{k} \cos (k \theta), \quad \hat{g}_{0}, \hat{g}_{1}, \ldots, \hat{g}_{m} \in \mathbb{R}, \quad m \in \mathbb{N},
$$

the $n$th Toeplitz matrix generated by $g$ is the real symmetric banded matrix given by

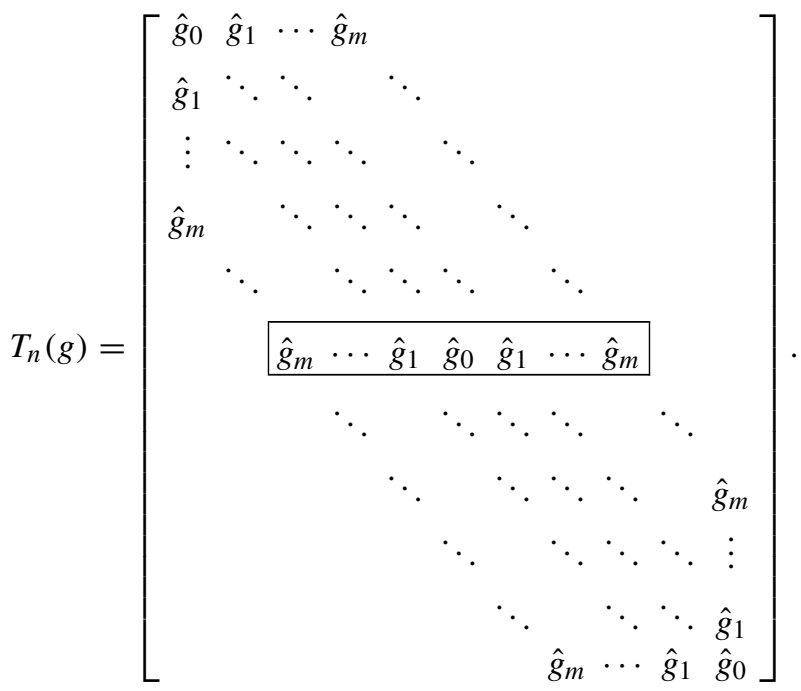


The numerical approximation of the eigenvalues of real symmetric banded Toeplitz matrices is a problem that has been faced by several authors; see, e.g., Arbenz [2], Badía and Vidal [3], Bini and Pan [5], the authors and Serra-Capizzano [13], and Trench [16-20]. Less attention has been devoted to the numerical approximation of the eigenvalues of preconditioned banded symmetric Toeplitz matrices of the form $T_{n}(u)^{-1} T_{n}(v)$, with $u, v$ being RCTPs. Yet, this problem is worthy of consideration as noted in [4, Section 1]. Some algorithms to solve it have been proposed in [1, 4]. For general discussions on the various algorithmic proposals for solving eigenvalue problems related to banded Toeplitz matrices, we refer the reader [2, Section 1] and [4, Section 1].

In this paper, we propose a new algorithm for the numerical approximation of the eigenvalues of preconditioned banded symmetric Toeplitz matrices. The algorithm relies on the following conjecture, which has been formulated by Serra-Capizzano in [1], on the basis of several numerical experiments.

Conjecture 1 Let $u, v$ be RCTPs, with $u>0$ on $(0, \pi)$, and suppose that $f=v / u$ is monotone increasing over $(0, \pi)$. Set $X_{n}=T_{n}(u)^{-1} T_{n}(v)$ for all $n$. Then, for every integer $\alpha \geq 0$, every $n$ and every $j=1, \ldots, n$, the following asymptotic expansion holds:

where:

$$
\lambda_{j}\left(X_{n}\right)=f\left(\theta_{j, n}\right)+\sum_{k=1}^{\alpha} c_{k}\left(\theta_{j, n}\right) h^{k}+E_{j, n, \alpha},
$$

- The eigenvalues of $X_{n}$ are arranged in non-decreasing order, $\lambda_{1}\left(X_{n}\right) \leq \ldots \leq$ $\lambda_{n}\left(X_{n}\right){ }^{1}$

- $\left\{c_{k}\right\}_{k=1,2, \ldots}$ is a sequence of functions from $(0, \pi)$ to $\mathbb{R}$ which depends only on $u, v$.

- $\quad h=\frac{1}{n+1}$ and $\theta_{j, n}=\frac{j \pi}{n+1}=j \pi h$.

- $E_{j, n, \alpha}=O\left(h^{\alpha+1}\right)$ is the remainder (the error), which satisfies the inequality $\left|E_{j, n, \alpha}\right| \leq C_{\alpha} h^{\alpha+1}$ for some constant $C_{\alpha}$ depending only on $\alpha, u, v$.

In the case where $u=1$ identically, Conjecture 1 was originally formulated and supported through numerical experiments in [13]. In the case where $u=1$ identically and $v$ satisfies some additional assumptions, Conjecture 1 was formally proved by Bogoya, Böttcher, Grudsky, and Maximenko in a sequence of recent papers $[6,8,10]$.

Assuming Conjecture 1, in Section 2 of this paper, we describe and analyze a new algorithm for computing the eigenvalues of $X_{n}=T_{n}(u)^{-1} T_{n}(v)$; and in Section 3, we illustrate its performance through numerical experiments. The algorithm, which is suited for parallel implementation and may be called matrix-less as it does not need to store the entries of $X_{n}$, combines the extrapolation procedure proposed in [1, 13] — which allows the computation of some of the eigenvalues of $X_{n}$-with an appropriate interpolation process, thus allowing the simultaneous computation of all the eigenvalues of $X_{n}$. In Section 4, we provide a generalization of the

\footnotetext{
${ }^{1}$ Note that the eigenvalues of $X_{n}$ are real, because $X_{n}$ is similar to the symmetric matrix $T_{n}(u)^{-1 / 2} T_{n}(v) T_{n}(u)^{-1 / 2}$.
} 
algorithm to the case where $f=v / u$ is non-monotone; this generalization is based on another conjecture which is analogous to Conjecture 1 and which will be discussed later on. In Section 5, we draw conclusions and suggest possible future lines of research.

\section{The algorithm}

Throughout this paper, we associate with each positive integer $n \in \mathbb{N}=\{1,2,3, \ldots\}$ the stepsize $h=\frac{1}{n+1}$ and the grid points $\theta_{j, n}=j \pi h, j=1, \ldots, n$. For notational convenience, we will always denote a positive integer and the associated stepsize in a similar way, in the sense that if the positive integer is denoted by $n$, the associated stepsize is denoted by $h$; if the positive integer is denoted by $n_{j}$, the associated stepsize is denoted by $h_{j}$; etc. Throughout this section, we make the following assumptions:

- $\quad u, v, f$ are as in Conjecture 1 .

- $\quad n, n_{1}, \alpha \in \mathbb{N}$ are fixed parameters and $X_{n}=T_{n}(u)^{-1} T_{n}(v)$.

- $n_{k}=2^{k-1}\left(n_{1}+1\right)-1$ for $k=2, \ldots, \alpha$.

- $j_{k}=2^{k-1} j_{1}$ for $j_{1}=1, \ldots, n_{1}$ and $k=2, \ldots, \alpha$. Note that $j_{k}=j_{k}\left(j_{1}\right)$ depends not only on $k$ but also on $j_{1}$, though we hide the dependence on $j_{1}$ for notational simplicity. Note also that $j_{k}$ is the index in $\left\{1, \ldots, n_{k}\right\}$ such that $\theta_{j_{k}, n_{k}}=\theta_{j_{1}, n_{1}}$. Hence, the grid $\left\{\theta_{j_{k}, n_{k}}: j_{1}=1, \ldots, n_{1}\right\}$ is the same as the grid $\left\{\theta_{j_{1}, n_{1}}: j_{1}=1, \ldots, n_{1}\right\}$ for all $k=2, \ldots, \alpha$.

A graphical representation of the grids $\left\{\theta_{1, n_{k}}, \ldots, \theta_{n_{k}, n_{k}}\right\}, k=1, \ldots, \alpha$, is reported in Fig. 1 for $n_{1}=5$ and $\alpha=4$. For each "level" $k=2, \ldots, \alpha$, the corresponding red circles highlight the subgrid $\left\{\theta_{j_{k}, n_{k}}: j_{1}=1, \ldots, n_{1}\right\}$ which coincides with the coarsest grid $\left\{\theta_{j_{1}, n_{1}}: j_{1}=1, \ldots, n_{1}\right\}$.

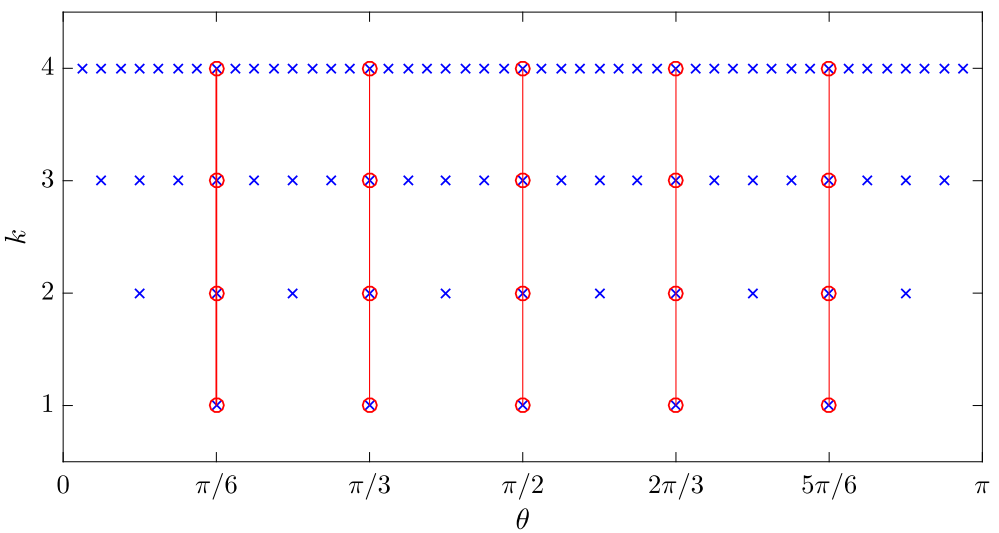

Fig. 1 Representation of the grids $\left\{\theta_{1, n_{k}}, \ldots, \theta_{n_{k}, n_{k}}\right\}, k=1, \ldots, \alpha$, for $n_{1}=5$ and $\alpha=4$ 


\subsection{Description and formulation of the algorithm}

The algorithm we are going to describe is designed for computing the eigenvalues of $X_{n}$ in the case where $n$ is large with respect to $n_{1}, \ldots, n_{\alpha}$, so that the computation of the eigenvalues of $X_{n}$ is hard from a computational viewpoint but the computation of the eigenvalues of $X_{n_{1}}, \ldots, X_{n_{\alpha}}$-which is required in the algorithm-can be efficiently performed by any standard eigensolver (e.g., MATLAB's eig function); see also Remark 1 below. The algorithm is composed of two phases: a first phase where we invoke extrapolation procedures from $[1,13]$ and a second phase where local interpolation techniques are employed.

Extrapolation For each fixed $j_{1}=1, \ldots, n_{1}$, we apply $\alpha$ times the expansion (1) with $n=n_{1}, n_{2}, \ldots, n_{\alpha}$ and $j=j_{1}, j_{2}, \ldots, j_{\alpha}$. Since $\theta_{j_{1}, n_{1}}=\theta_{j_{2}, n_{2}}=\ldots=\theta_{j_{\alpha}, n_{\alpha}}$ (by definition of $j_{2}, \ldots, j_{\alpha}$ ), we obtain

$$
\left\{\begin{array}{l}
E_{j_{1}, n_{1}, 0}=c_{1}\left(\theta_{j_{1}, n_{1}}\right) h_{1}+c_{2}\left(\theta_{j_{1}, n_{1}}\right) h_{1}^{2}+\ldots+c_{\alpha}\left(\theta_{j_{1}, n_{1}}\right) h_{1}^{\alpha}+E_{j_{1}, n_{1}, \alpha} \\
E_{j_{2}, n_{2}, 0}=c_{1}\left(\theta_{j_{1}, n_{1}}\right) h_{2}+c_{2}\left(\theta_{j_{1}, n_{1}}\right) h_{2}^{2}+\ldots+c_{\alpha}\left(\theta_{j_{1}, n_{1}}\right) h_{2}^{\alpha}+E_{j_{2}, n_{2}, \alpha} \\
\vdots \\
E_{j_{\alpha}, n_{\alpha}, 0}=c_{1}\left(\theta_{j_{1}, n_{1}}\right) h_{\alpha}+c_{2}\left(\theta_{j_{1}, n_{1}}\right) h_{\alpha}^{2}+\ldots+c_{\alpha}\left(\theta_{j_{1}, n_{1}}\right) h_{\alpha}^{\alpha}+E_{j_{\alpha}, n_{\alpha}, \alpha}
\end{array}\right.
$$

where

$$
E_{j_{k}, n_{k}, 0}=\lambda_{j_{k}}\left(X_{n_{k}}\right)-f\left(\theta_{j_{1}, n_{1}}\right), \quad k=1, \ldots, \alpha,
$$

and

$$
\left|E_{j_{k}, n_{k}, \alpha}\right| \leq C_{\alpha} h_{k}^{\alpha+1}, \quad k=1, \ldots, \alpha .
$$

Let $\tilde{c}_{1}\left(\theta_{j_{1}, n_{1}}\right), \ldots, \tilde{c}_{\alpha}\left(\theta_{j_{1}, n_{1}}\right)$ be the approximations of $c_{1}\left(\theta_{j_{1}, n_{1}}\right), \ldots, c_{\alpha}\left(\theta_{j_{1}, n_{1}}\right)$ obtained by removing all the errors $E_{j_{1}, n_{1}, \alpha}, \ldots, E_{j_{\alpha}, n_{\alpha}, \alpha}$ in (2) and by solving the resulting linear system:

$$
\left\{\begin{array}{l}
E_{j_{1}, n_{1}, 0}=\tilde{c}_{1}\left(\theta_{j_{1}, n_{1}}\right) h_{1}+\tilde{c}_{2}\left(\theta_{j_{1}, n_{1}}\right) h_{1}^{2}+\ldots+\tilde{c}_{\alpha}\left(\theta_{j_{1}, n_{1}}\right) h_{1}^{\alpha} \\
E_{j_{2}, n_{2}, 0}=\tilde{c}_{1}\left(\theta_{j_{1}, n_{1}}\right) h_{2}+\tilde{c}_{2}\left(\theta_{j_{1}, n_{1}}\right) h_{2}^{2}+\ldots+\tilde{c}_{\alpha}\left(\theta_{j_{1}, n_{1}}\right) h_{2}^{\alpha} \\
\vdots \\
E_{j_{\alpha}, n_{\alpha}, 0}=\tilde{c}_{1}\left(\theta_{j_{1}, n_{1}}\right) h_{\alpha}+\tilde{c}_{2}\left(\theta_{j_{1}, n_{1}}\right) h_{\alpha}^{2}+\ldots+\tilde{c}_{\alpha}\left(\theta_{j_{1}, n_{1}}\right) h_{\alpha}^{\alpha}
\end{array}\right.
$$

Note that this way of computing approximations for $c_{1}\left(\theta_{j_{1}, n_{1}}\right), \ldots, c_{\alpha}\left(\theta_{j_{1}, n_{1}}\right)$ was already proposed in [1,13], and it is completely analogous to the Richardson extrapolation procedure that is employed in the context of Romberg integration to accelerate the convergence of the trapezoidal rule [15, Section 3.4]. In this regard, the asymptotic expansion (1) plays here the same role as the Euler-Maclaurin summation formula [15, Section 3.3]. For more advanced studies on extrapolation methods, we refer the reader to [11]. The next theorem shows that the approximation error $\left|c_{k}\left(\theta_{j_{1}, n_{1}}\right)-\tilde{c}_{k}\left(\theta_{j_{1}, n_{1}}\right)\right|$ is $O\left(h_{1}^{\alpha-k+1}\right)$. 
Theorem 1 There exists a constant $A_{\alpha}$ depending only on $\alpha, u, v$ such that, for $j_{1}=$ $1, \ldots, n_{1}$ and $k=1, \ldots, \alpha$,

$$
\left|c_{k}\left(\theta_{j_{1}, n_{1}}\right)-\tilde{c}_{k}\left(\theta_{j_{1}, n_{1}}\right)\right| \leq A_{\alpha} h_{1}^{\alpha-k+1} .
$$

\section{Proof See Appendix A.}

Interpolation Fix an index $j \in\{1, \ldots, n\}$. To compute an approximation of $\lambda_{j}\left(X_{n}\right)$ through the expansion (1), we would need the value $c_{k}\left(\theta_{j, n}\right)$ for each $k=1, \ldots, \alpha$. Of course, $c_{k}\left(\theta_{j, n}\right)$ is not available in practice, but we can approximate it by interpolating in some way the values $\tilde{c}_{k}\left(\theta_{j_{1}, n_{1}}\right), j_{1}=1, \ldots, n_{1}$. For example, we may define $\tilde{c}_{k}(\theta)$ as the interpolation polynomial of the data $\left(\theta_{1, n_{1}}, \tilde{c}_{k}\left(\theta_{1, n_{1}}\right)\right), \ldots,\left(\theta_{n_{1}, n_{1}}, \tilde{c}_{k}\left(\theta_{n_{1}, n_{1}}\right)\right)$-so that $\tilde{c}_{k}(\theta)$ is expected to be an approximation of $c_{k}(\theta)$ over the whole interval $(0, \pi)$-and take $\tilde{c}_{k}\left(\theta_{j, n}\right)$ as an approximation to $c_{k}\left(\theta_{j, n}\right)$. It is known, however, that interpolation over a large number of uniform nodes is not advisable as it may give rise to spurious oscillations (Runge's phenomenon [12, p. 78]). It is therefore better to adopt another kind of approximation. An alternative could be the following: we approximate $c_{k}(\theta)$ by the spline function $\tilde{c}_{k}(\theta)$ which is linear on each interval $\left[\theta_{j_{1}, n_{1}}, \theta_{j_{1}+1, n_{1}}\right]$ and takes the value $\tilde{c}_{k}\left(\theta_{j_{1}, n_{1}}\right)$ at $\theta_{j_{1}, n_{1}}$ for all $j_{1}=1, \ldots, n_{1}$. This strategy removes for sure any spurious oscillation, yet it is not accurate. In particular, it does not preserve the accuracy of approximation at the nodes $\theta_{j_{1}, n_{1}}$ established in Theorem 1 , i.e., there is no guarantee that $\left|c_{k}(\theta)-\tilde{c}_{k}(\theta)\right| \leq B_{\alpha} h_{1}^{\alpha-k+1}$ for $\theta \in(0, \pi)$ or $\left|c_{k}\left(\theta_{j, n}\right)-\tilde{c}_{k}\left(\theta_{j, n}\right)\right| \leq B_{\alpha} h_{1}^{\alpha-k+1}$ for $j=1, \ldots, n$, with $B_{\alpha}$ being a constant depending only on $\alpha, u, v$. As proved in Theorem 2, a local approximation strategy that preserves the accuracy (5), at least if $c_{k}(\theta)$ is sufficiently smooth, is the following: let $\theta^{(1)}, \ldots, \theta^{(\alpha-k+1)}$ be $\alpha-k+1$ points of the grid $\left\{\theta_{1, n_{1}}, \ldots, \theta_{n_{1}, n_{1}}\right\}$ which are closest to the point $\theta_{j, n},{ }^{2}$ and let $\tilde{c}_{k, j}(\theta)$ be the interpolation polynomial of the data $\left(\theta^{(1)}, \tilde{c}_{k}\left(\theta^{(1)}\right)\right), \ldots,\left(\theta^{(\alpha-k+1)}, \tilde{c}_{k}\left(\theta^{(\alpha-k+1)}\right)\right)$; then, we approximate $c_{k}\left(\theta_{j, n}\right)$ by $\tilde{c}_{k, j}\left(\theta_{j, n}\right)$. Note that, by selecting $\alpha-k+1$ points from $\left\{\theta_{1, n_{1}}, \ldots, \theta_{n_{1}, n_{1}}\right\}$, we are implicitly assuming that $n_{1} \geq \alpha-k+1$.

Theorem 2 Let $1 \leq k \leq \alpha$, and suppose $n_{1} \geq \alpha-k+1$ and $c_{k} \in C^{\alpha-k+1}([0, \pi])$. For $j=1, \ldots, n$, if $\theta^{(1)}, \ldots, \theta^{(\alpha-k+1)}$ are $\alpha-k+1$ points of $\left\{\theta_{1, n_{1}}, \ldots, \theta_{n_{1}, n_{1}}\right\}$ which are closest to $\theta_{j, n}$, and if $\tilde{c}_{k, j}(\theta)$ is the interpolation polynomial of the data $\left(\theta^{(1)}, \tilde{c}_{k}\left(\theta^{(1)}\right)\right), \ldots,\left(\theta^{(\alpha-k+1)}, \tilde{c}_{k}\left(\theta^{(\alpha-k+1)}\right)\right)$, then

$$
\left|c_{k}\left(\theta_{j, n}\right)-\tilde{c}_{k, j}\left(\theta_{j, n}\right)\right| \leq B_{\alpha} h_{1}^{\alpha-k+1}
$$

for some constant $B_{\alpha}$ depending only on $\alpha, u, v$.

Proof See Appendix A.

\footnotetext{
${ }^{2}$ These $\alpha-k+1$ points are uniquely determined by $\theta_{j, n}$ except in the case where $\theta_{j, n}$ coincides with either a grid point $\theta_{j_{1}, n_{1}}$ or the midpoint between two consecutive grid points $\theta_{j_{1}, n_{1}}$ and $\theta_{j_{1}+1, n_{1}}$.
} 
Formulation of the algorithm We are now ready to formulate our algorithm for computing the eigenvalues of $X_{n}$. As we shall see in Remark 4, the algorithm is suited for parallel implementation. Since it does not even need to store the entries of $X_{n}$, it may be called matrix-less. It can be used for computing either a specific eigenvalue $\lambda_{j}\left(X_{n}\right)$, a subset of the eigenvalues of $X_{n}$, or the whole spectrum of $X_{n}$. A plain (non-parallel) MATLAB implementation of this algorithm is reported in Appendix B.

Algorithm 1 Given two RCTPs $u$, $v$ (with $u>0$ on $(0, \pi)$ and $f=v / u$ monotone increasing over $(0, \pi)$ as in Conjecture 1$)$, three integers $n, n_{1}, \alpha \in \mathbb{N}$ with $n_{1} \geq \alpha$, and $S \subseteq\{1, \ldots, n\}$, we compute an approximation of the eigenvalues $\left\{\lambda_{j}\left(X_{n}\right): j \in\right.$ $S\}$ as follows:

1. For $j_{1}=1, \ldots, n_{1}$ compute $\tilde{c}_{1}\left(\theta_{j_{1}, n_{1}}\right), \ldots, \tilde{c}_{\alpha}\left(\theta_{j_{1}, n_{1}}\right)$ by solving (4)

2. For $j \in S$

- For $k=1, \ldots, \alpha$

- Determine $\alpha-k+1$ points $\theta^{(1)}, \ldots, \theta^{(\alpha-k+1)} \in\left\{\theta_{1, n_{1}}, \ldots, \theta_{n_{1}, n_{1}}\right\}$ which are closest to $\theta_{j, n}$

- Compute $\tilde{c}_{k, j}\left(\theta_{j, n}\right)$, where $\tilde{c}_{k, j}(\theta)$ is the interpolation polynomial of $\left(\theta^{(1)}, \tilde{c}_{k}\left(\theta^{(1)}\right)\right), \ldots,\left(\theta^{(\alpha-k+1)}, \tilde{c}_{k}\left(\theta^{(\alpha-k+1)}\right)\right)$

- Compute $\tilde{\lambda}_{j}\left(X_{n}\right)=f\left(\theta_{j, n}\right)+\sum_{k=1}^{\alpha} \tilde{c}_{k, j}\left(\theta_{j, n}\right) h^{k}$

3. Return $\left\{\tilde{\lambda}_{j}\left(X_{n}\right): j \in S\right\}$ as an approximation to $\left\{\lambda_{j}\left(X_{n}\right): j \in S\right\}$

Remark 1 Algorithm 1 is specifically designed for computing the eigenvalues of $X_{n}$ in the case where the matrix size $n$ is quite large. When applying this algorithm, it is implicitly assumed that $n_{1}$ and $\alpha$ are small (much smaller than $n$ ), so that each $n_{k}=2^{k-1}\left(n_{1}+1\right)-1$ is small as well and the computation of the eigenvalues of $X_{n_{k}}$-which is required in the first step — can be efficiently performed by any standard eigensolver (e.g., MATLAB's eig function).

Remark 2 A careful evaluation shows that the computational cost of Algorithm 1 is bounded by

$$
C\left(\alpha^{2} n_{1}+\alpha^{3}|S|\right)+\sum_{k=1}^{\alpha} C_{\text {eig }}\left(n_{k}\right),
$$

where $|S|$ is the cardinality of $S, C$ is a constant depending only on $f$, and $C_{\text {eig }}\left(n_{k}\right)$ is the cost for computing the eigenvalues of $X_{n_{k}}$.

Remark 3 Algorithm 1 can be optimized in several ways. For example, if $S=\{j\}$, so that only the $j$ th eigenvalue $\lambda_{j}\left(X_{n}\right)$ must be computed, then in the first step one can just compute the values $\tilde{c}_{1}\left(\theta_{j_{1}, n_{1}}\right), \ldots, \tilde{c}_{\alpha}\left(\theta_{j_{1}, n_{1}}\right)$ for $\theta_{j_{1}, n_{1}} \in\left\{\theta^{(1)}, \ldots, \theta^{(\alpha)}\right\}$, where $\theta^{(1)}, \ldots, \theta^{(\alpha)}$ are $\alpha$ points in $\left\{\theta_{1, n_{1}}, \ldots, \theta_{n_{1}, n_{1}}\right\}$ which are closest to $\theta_{j, n}$. Indeed, only these values are needed in the second step. A similar consideration applies in the case where only the extremal eigenvalues of $X_{n}$ must be computed, and also in the case where $S$ is a small subset of $\{1, \ldots, n\}$ of the form $\{j, \ldots, j+r\}$, with $r \ll n$. 
Remark 4 Suppose $|S|=n$ and consider the ideal situation where we have $n$ processors. Then, the $j$ th processor can compute the $j$ th eigenvalue $\lambda_{j}\left(X_{n}\right)$ independently of the others. In view of Remark 3 , the $j$ th processor can act as follows:

- In the first step of the algorithm, it computes only the values $\tilde{c}_{1}\left(\theta_{j_{1}, n_{1}}\right), \ldots, \tilde{c}_{\alpha}\left(\theta_{j_{1}, n_{1}}\right)$ for $\theta_{j_{1}, n_{1}} \in\left\{\theta^{(1)}, \ldots, \theta^{(\alpha)}\right\}$, where $\theta^{(1)}, \ldots, \theta^{(\alpha)}$ are $\alpha$ points in $\left\{\theta_{1, n_{1}}, \ldots, \theta_{n_{1}, n_{1}}\right\}$ which are closest to $\theta_{j, n}$.

- It performs the second step of the algorithm for the index $j$ only.

It is clear that such a parallel implementation is very fast as the computation of all the eigenvalues of $X_{n}$ takes the same time as the computation of one eigenvalue only. A similar consideration also applies in the case where $|S|<n$ and we have $|S|$ processors, each of which has to compute only one of the requested $|S|$ eigenvalues. In a more realistic situation, we will not have a number of processors equal to $|S|$ if $|S|$ is large. Instead, we will have $p$ processors with $p \ll|S|$. In this case, we can divide $S$ into $p$ different subsets $S_{1}, \ldots, S_{p}$ of approximately the same cardinality and assign to the $i$ th processor the computation of the eigenvalues corresponding to $S_{i}, i=1, \ldots, p$. When doing so, it is advisable that each $S_{i}$ is constructed so that the "positions" $\theta_{j, n}$ of the related eigenvalues $\lambda_{j}\left(X_{n}\right)$ are close to each other, because in this way each processor will have the possibility to perform a reduced form of the first step of the algorithm, in analogy with what has been explained above for the case $p=|S|$. For example, if $|S|=n$ and $n$ is a multiple of $p$, then we can assign to the $i$ th processor the computation of the eigenvalues $\lambda_{j}\left(X_{n}\right)$ for $j=(i-1)(n / p)+1, \ldots, i(n / p)$, so that in the first step of the algorithm the $i$ th processor will only have to compute $\tilde{c}_{1}\left(\theta_{j_{1}, n_{1}}\right), \ldots, \tilde{c}_{\alpha}\left(\theta_{j_{1}, n_{1}}\right)$ for $\theta_{j_{1}, n_{1}}$ in a neighborhood of the interval $\left[\theta_{(i-1)(n / p)+1, n}, \theta_{i(n / p), n}\right]$.

\subsection{Error estimate}

Theorem 3 Assume that Conjecture 1 holds. Suppose $n \geq n_{1} \geq \alpha$ and $c_{k} \in$ $C^{\alpha-k+1}([0, \pi])$ for $k=1, \ldots, \alpha$. Let $\left(\tilde{\lambda}_{1}\left(X_{n}\right), \ldots, \tilde{\lambda}_{n}\left(X_{n}\right)\right)$ be the approximation of $\left(\lambda_{1}\left(X_{n}\right), \ldots, \lambda_{n}\left(X_{n}\right)\right)$ computed by Algorithm 1 . Then, there exists a constant $D_{\alpha}$ depending only on $\alpha, u, v$ such that, for $j=1, \ldots, n$,

$$
\left|\lambda_{j}\left(X_{n}\right)-\tilde{\lambda}_{j}\left(X_{n}\right)\right| \leq D_{\alpha} h_{1}^{\alpha} h \text {. }
$$

Proof By (1) and Theorem 2,

$$
\begin{aligned}
\left|\lambda_{j}\left(X_{n}\right)-\tilde{\lambda}_{j}\left(X_{n}\right)\right| & =\left|f\left(\theta_{j, n}\right)+\sum_{k=1}^{\alpha} c_{k}\left(\theta_{j, n}\right) h^{k}+E_{j, n, \alpha}-f\left(\theta_{j, n}\right)-\sum_{k=1}^{\alpha} \tilde{c}_{k, j}\left(\theta_{j, n}\right) h^{k}\right| \\
& =\left|\sum_{k=1}^{\alpha}\left(c_{k}\left(\theta_{j, n}\right)-\tilde{c}_{k, j}\left(\theta_{j, n}\right)\right) h^{k}+E_{j, n, \alpha}\right| \\
& \leq B_{\alpha} \sum_{k=1}^{\alpha} h_{1}^{\alpha-k+1} h^{k}+C_{\alpha} h^{\alpha+1} \leq D_{\alpha} h_{1}^{\alpha} h,
\end{aligned}
$$

where $D_{\alpha}=(\alpha+1) \max \left(B_{\alpha}, C_{\alpha}\right)$. 
Remark 5 The error estimate provided in Theorem 3 suggests that the eigenvalue approximations provided by Algorithm 1 improve as $n$ increases, i.e., as $h$ decreases. Numerical experiments reveal that this is in fact the case (see Example 2 below).

Remark 6 Theorem 3 shows that, for any fixed $\alpha \geq 1$, the numerical eigenvalues computed by Algorithm 1 converge like $h_{1}^{\alpha}$ to the exact eigenvalues as $n_{1}$ grows. In practice, it is advisable to fix $\alpha$ and increase $n_{1}$ until a proper stopping criterion is reached. The other way (fix $n_{1}$ and increase $\alpha$ ) is not advisable as the constant $D_{\alpha}$ in Theorem 3 apparently grows very quickly with $\alpha$ (see Example 1 below) and, consequently, there is no guarantee on the convergence of the algorithm as $\alpha$ grows (see Example 5 below).

\section{Numerical experiments}

In this section, we illustrate through numerical examples the performance of Algorithm 1. Numerical experiments have been performed with MATLAB R2015b (64 bit) on a platform with 4GB RAM, using an Intel ${ }^{\circledR}$ Celeron ${ }^{\circledR}$ Processor N2820 (up to $2.39 \mathrm{GHz}, 1 \mathrm{MB} \mathrm{L} 2$ cache). The CPU times for Algorithm 1 refer to the plain MATLAB implementation reported in Appendix B. In what follows, the symbol $\varepsilon_{j, n}$ denotes the error $\left|\lambda_{j}\left(X_{n}\right)-\tilde{\lambda}_{j}\left(X_{n}\right)\right|$, which occurs when approximating the exact eigenvalue $\lambda_{j}\left(X_{n}\right)$ with the corresponding numerical eigenvalue $\tilde{\lambda}_{j}\left(X_{n}\right)$ computed by Algorithm 1 . The inputs $u, v, n, n_{1}, \alpha$ with which Algorithm 1 is applied are specified in each example.

Example 1 Let

$$
\begin{aligned}
& u(\theta)=1, \\
& v(\theta)=6-8 \cos (\theta)+2 \cos (2 \theta) .
\end{aligned}
$$

Note that $f(\theta)=v(\theta) / u(\theta)=v(\theta)$ is monotone increasing on $(0, \pi)$. Suppose we want to approximate the eigenvalues of $X_{n}=T_{n}(u)^{-1} T_{n}(v)=T_{n}(f)$ for $n=5000$. Let $\tilde{\lambda}_{j}\left(X_{n}\right)$ be the approximation of $\lambda_{j}\left(X_{n}\right)$ obtained by applying Algorithm 1 with $n_{1}=10$ and $\alpha=7$. In Fig. 2, we plot the errors $\varepsilon_{j, n}$ versus $\theta_{j, n}$ for $j=$ $1, \ldots, n$. We note that the largest errors are attained when either $\theta_{j, n} \approx 0$ or $\theta_{j, n} \approx \pi$. As highlighted also in Example 3 below, this is probably due to two concomitant factors:

- The errors $\varepsilon_{j, n}$ are supposed to be smaller for $\theta_{j, n} \in\left[\theta_{1, n_{1}}, \theta_{n_{1}, n_{1}}\right]=$ $[\pi / 11,10 \pi / 11]$, because in this case the approximations $\tilde{c}_{k, j}\left(\theta_{j, n}\right)$ computed by Algorithm 1 for the values $c_{k}\left(\theta_{j, n}\right)$ are expected to be more accurate as the interpolation polynomial $\tilde{c}_{k, j}(\theta)$ is evaluated inside the convex hull of the interpolation nodes.

- $\theta=0$ and $\theta=\pi$ are the two points on $[0, \pi]$ where $f^{\prime}$ vanishes, which means that the monotonicity of $f$ is "weak" around these points (recall that Algorithm 1 works under the assumption that $f$ is monotone as in Conjecture 1). 


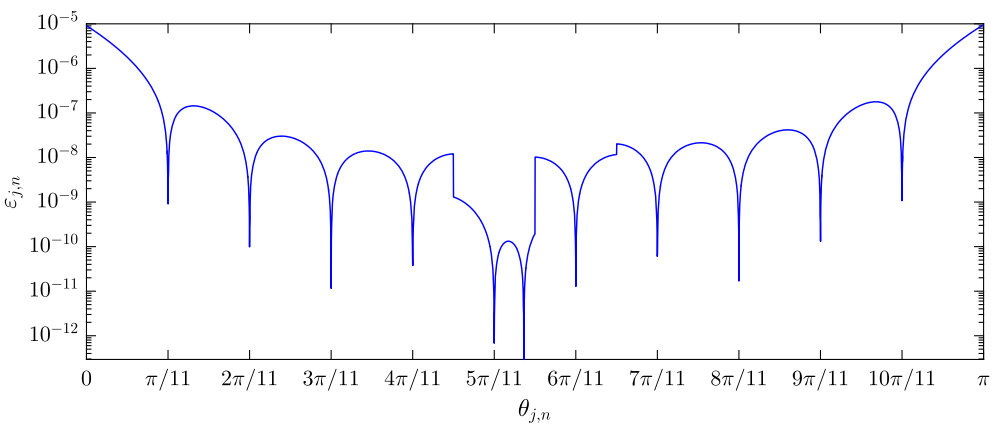

Fig. 2 Example 1: errors $\varepsilon_{j, n}$ versus $\theta_{j, n}$ for $j=1, \ldots, n$ in the case where $u(\theta)=1, v(\theta)=6-$ $8 \cos (\theta)+2 \cos (2 \theta), n=5000, n_{1}=10$, and $\alpha=7$

In reference to the previous discussion, we note that the maximum error for $\theta_{j, n} \in$ $\left[\theta_{1, n_{1}}, \theta_{n_{1}, n_{1}}\right]$ is given by

$$
\max \left\{\varepsilon_{j, n}: \theta_{j, n} \in\left[\theta_{1, n_{1}}, \theta_{n_{1}, n_{1}}\right]\right\} \approx 1.7803 \cdot 10^{-7}
$$

which is about two order of magnitude less than

$$
\max _{j=1, \ldots, n} \varepsilon_{j, n} \approx 9.5167 \cdot 10^{-6}
$$

A careful look at Fig. 2 shows that, aside from the exceptional minimum attained inside the interval $(5 \pi / 11,6 \pi / 11)$, the local minima of $\varepsilon_{j, n}$ are attained when $\theta_{j, n}$ is approximately equal to some of the grid points $\theta_{j_{1}, n_{1}}, j_{1}=1, \ldots, n_{1}$. This is no surprise, because for $\theta_{j, n}=\theta_{j_{1}, n_{1}}$ we have $\tilde{c}_{k, j}\left(\theta_{j, n}\right)=\tilde{c}_{k}\left(\theta_{j_{1}, n_{1}}\right)$ and $c_{k}\left(\theta_{j, n}\right)=$ $c_{k}\left(\theta_{j_{1}, n_{1}}\right)$, which means that the error of the approximation $\tilde{c}_{k, j}\left(\theta_{j, n}\right) \approx c_{k}\left(\theta_{j, n}\right)$ reduces to the error of the approximation $\tilde{c}_{k}\left(\theta_{j_{1}, n_{1}}\right) \approx c_{k}\left(\theta_{j_{1}, n_{1}}\right)$; that is, we are not introducing further error due to the interpolation process. To conclude, we make the following observation: for $\alpha, u, v$ as in this example, Theorem 3 yields

$$
D_{\alpha} \geq \frac{\max _{j=1, \ldots, n} \varepsilon_{j, n}}{h_{1}^{\alpha} h} \approx 9.2745 \cdot 10^{5}>\alpha^{\alpha}=8.23543 \cdot 10^{5} .
$$

This suggests that, unfortunately, the best constant $D_{\alpha}$ for which the error estimate of Theorem 3 is satisfied grows very quickly with $\alpha$.

Example 2 Let $u, v, f$ be as in Example 1. Suppose we want to approximate the eigenvalues of $X_{n}=T_{n}(u)^{-1} T_{n}(v)=T_{n}(f)$ for $n=10000$. Let $\tilde{\lambda}_{j}\left(X_{n}\right)$ be the approximation of $\lambda_{j}\left(X_{n}\right)$ obtained by applying Algorithm 1 with $n_{1}=10$ and $\alpha=7$ as in Example 1. In Fig. 3, we plot the errors $\varepsilon_{j, n}$ versus $\theta_{j, n}$ for $j=1, \ldots, n$. We note that the errors in Fig. 3 are smaller than in Fig. 2. This shows that the eigenvalue approximations provided by Algorithm 1 improve as $n$ increases (see also Remark 5). 


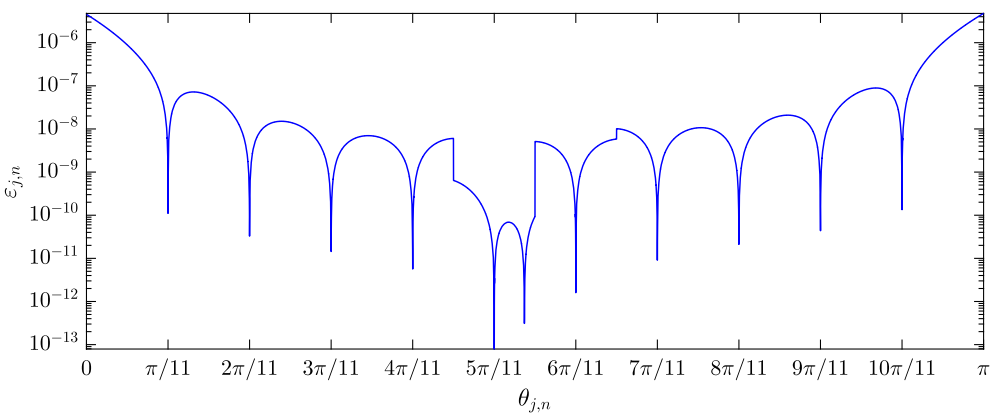

Fig. 3 Example 2: errors $\varepsilon_{j, n}$ versus $\theta_{j, n}$ for $j=1, \ldots, n$ in the case where $u(\theta)=1, v(\theta)=6-$ $8 \cos (\theta)+2 \cos (2 \theta), n=10000, n_{1}=10$, and $\alpha=7$

Example 3 Let

$$
\begin{aligned}
& u(\theta)=1 \\
& v(\theta)=-\frac{1}{4}-\frac{1}{2} \cos (\theta)+\frac{1}{4} \cos (2 \theta)-\frac{1}{12} \cos (3 \theta) .
\end{aligned}
$$

Note that $f(\theta)=v(\theta) / u(\theta)=v(\theta)$ is monotone increasing on $(0, \pi)$. Suppose we want to approximate the eigenvalues of $X_{n}=T_{n}(u)^{-1} T_{n}(v)=T_{n}(f)$ for $n=10000$. Let $\tilde{\lambda}_{j}^{(m)}\left(X_{n}\right)$ be the approximation of $\lambda_{j}\left(X_{n}\right)$ obtained by applying Algorithm 1 with $n_{1}=10 \cdot 2^{m-1}$ and $\alpha=5$. In Fig. 4, we plot the errors $\varepsilon_{j, n}^{(m)}=\left|\lambda_{j}\left(X_{n}\right)-\tilde{\lambda}_{j}^{(m)}\left(X_{n}\right)\right|$ versus $\theta_{j, n}$ for $j=1, \ldots, n$ and $m=1,2,3,4$. We see from the figure that, as $m$ increases, the error decreases rather quickly everywhere except in a neighborhood of the point $\theta=\pi / 3$ where $f^{\prime}$ vanishes. Actually, the three points of $[0, \pi]$ where $f^{\prime}$ vanishes are $0, \pi / 3, \pi$, and these are precisely the points around which the error is higher than elsewhere. We remark that, as in Examples 1 and 2, the error $\varepsilon_{j, n}^{(m)}$ attains its local minima when $\theta_{j, n}$ is approximately equal to some of the nodes $\theta_{1, n_{1}}, \ldots, \theta_{n_{1}, n_{1}}$.

\section{Example 4 Let}

$$
\begin{aligned}
& u(\theta)=1, \\
& v(\theta)=\frac{301}{400}-\cos (\theta)+\frac{1}{5} \cos (2 \theta)+\frac{1}{10} \cos (3 \theta)-\frac{1}{20} \cos (4 \theta)+\frac{1}{400} \cos (6 \theta) .
\end{aligned}
$$

Note that $f(\theta)=v(\theta) / u(\theta)=v(\theta)$ is monotone increasing on $(0, \pi)$ and $f^{\prime}(\theta)=0$ only for $\theta=0, \pi{ }^{3}$ Suppose we want to approximate the eigenvalues of $X_{n}=T_{n}(u)^{-1} T_{n}(v)=T_{n}(f)$ for $n=10000$. Let $\tilde{\lambda}_{j}^{(m)}\left(X_{n}\right)$ be the approximation of $\lambda_{j}\left(X_{n}\right)$ obtained by applying Algorithm 1 with $n_{1}=25 \cdot 2^{m-1}$ and $\alpha=5$. In Fig. 5, we plot the errors $\varepsilon_{j, n}^{(m)}$ versus $\theta_{j, n}$ for $j=1, \ldots, n$ and $m=1,2,3,4$. Considerations analogous to those of Example 3 apply also in this case.

\footnotetext{
${ }^{3}$ Note that we always have $g^{\prime}(0)=g^{\prime}(\pi)=0$ whenever $g(\theta)$ is an RCTP.
} 

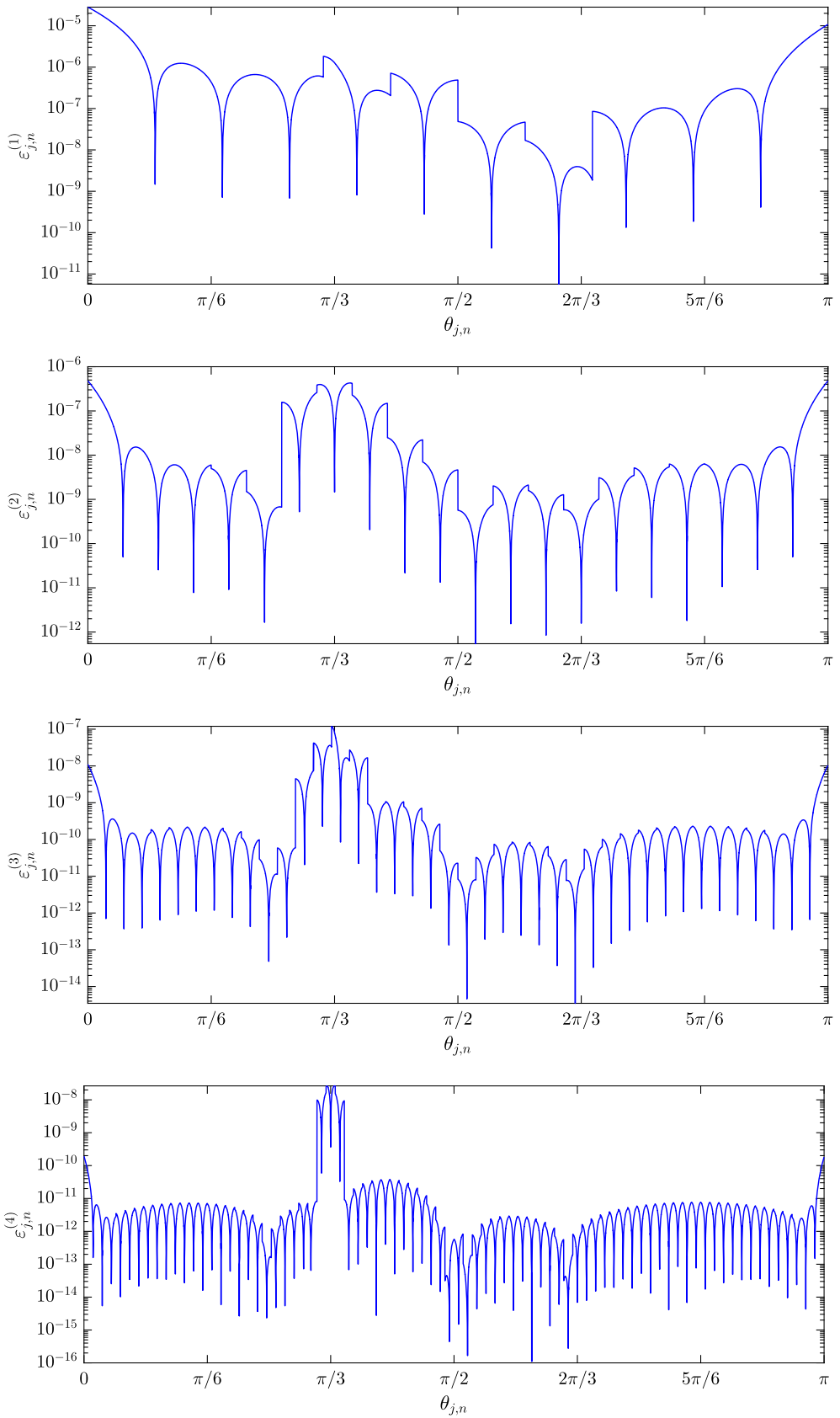

Fig. 4 Example 3: errors $\varepsilon_{j, n}^{(m)}$ versus $\theta_{j, n}$ for $j=1, \ldots, n$, in the case where $u(\theta)=1, v(\theta)=-\frac{1}{4}-$ $\frac{1}{2} \cos (\theta)+\frac{1}{4} \cos (2 \theta)-\frac{1}{12} \cos (3 \theta), n=10000, n_{1}=10 \cdot 2^{m-1}$, and $\alpha=5$ 

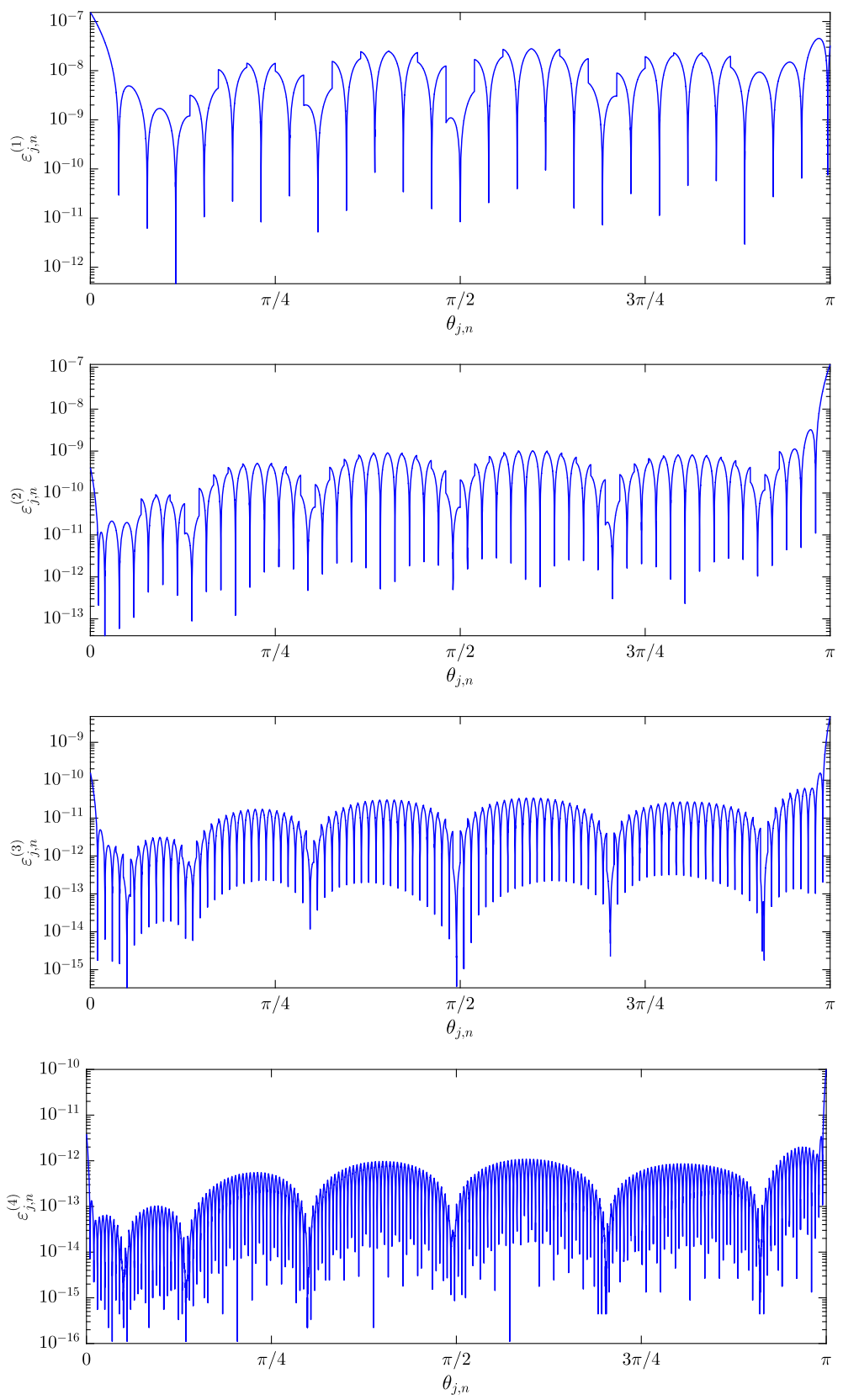

Fig. 5 Example 4: errors $\varepsilon_{j, n}^{(m)}$ versus $\theta_{j, n}$ for $j=1, \ldots, n$, in the case where $u(\theta)=1, v(\theta)=\frac{301}{400}-$ $\cos (\theta)+\frac{1}{5} \cos (2 \theta)+\frac{1}{10} \cos (3 \theta)-\frac{1}{20} \cos (4 \theta)+\frac{1}{400} \cos (6 \theta), n=10000, n_{1}=25 \cdot 2^{m-1}$, and $\alpha=5$ 
Example 5 Let $u, v, f$ as in Example 4. Suppose we want to approximate the eigenvalues of $X_{n}=T_{n}(u)^{-1} T_{n}(v)=T_{n}(f)$ for $n=10000$. Let $\tilde{\lambda}_{j}^{(m)}\left(X_{n}\right)$ be the approximation of $\lambda_{j}\left(X_{n}\right)$ obtained by applying Algorithm 1 with $n_{1}=25$ and $\alpha=4+m$. In Fig. 6 , we plot the errors $\varepsilon_{j, n}^{(m)}$ versus $\theta_{j, n}$ for $j=1, \ldots, n$ and $m=1,2,3,4$. By comparing Fig. 5 with Fig. 6, we see that the strategy of keeping $n_{1}$ fixed and increasing $\alpha$ is much less efficient than the strategy of keeping $\alpha$ fixed and increasing $n_{1}$. Indeed, while in Fig. 5 the error $\varepsilon_{j, n}^{(m)}$ decreases approximately in a uniform way by one order of magnitude as $m$ increases, this is not observed in Fig. 6. Note also that the computational cost of Algorithm 1 for $n_{1}=25 \cdot 2^{m-1}$ and $\alpha=5$ (as in Fig. 5) is essentially the same as the cost of Algorithm 1 for $n_{1}=25$ and $\alpha=4+m$ (as in Fig. 6), because the main task of the algorithm in both cases is the computation of the eigenvalues of $X_{n_{\alpha}}$, and in both cases $n_{\alpha}$ is approximately equal to $25 \cdot 2^{m+3}$. The bad behavior of Algorithm 1 when increasing $\alpha$ finds an explanation in the fact that, as observed in Example 1, the constant $D_{\alpha}$ appearing in the error estimate of Theorem 3 apparently grows very quickly with $\alpha$.

\section{Example 6 Let}

$$
\begin{aligned}
& u(\theta)=3+2 \cos (\theta), \\
& v(\theta)=2-\cos (\theta)-\cos (2 \theta) .
\end{aligned}
$$

Note that $f(\theta)=v(\theta) / u(\theta)=1-\cos (\theta)$ is monotone increasing on $(0, \pi)$ and $f^{\prime}(\theta)=0$ only for $\theta=0, \pi$. Suppose we want to approximate the eigenvalues of $X_{n}=T_{n}(u)^{-1} T_{n}(v)$ for $n=5000$. Let $\tilde{\lambda}_{j}^{(m)}\left(X_{n}\right)$ be the approximation of $\lambda_{j}\left(X_{n}\right)$ obtained by applying Algorithm 1 with $n_{1}=50 \cdot 2^{m-1}$ and $\alpha=4$. The graph of the errors $\varepsilon_{j, n}^{(m)}$ versus $\theta_{j, n}$ is shown in Fig. 7 for $j=1, \ldots, n$ and $m=1,2,3,4$. Table 1 compares the CPU times for computing the eigenvalues of $X_{n}$ by using MATLAB's eig function and Algorithm 1.

Example 7 This example is suggested by the cubic B-spline isogeometric analysis discretization of second-order eigenvalue problems [14, Section 10.7.3]. Let

$$
\begin{aligned}
& u(\theta)=1208+1191 \cos (\theta)+120 \cos (2 \theta)+\cos (3 \theta), \\
& v(\theta)=40-15 \cos (\theta)-24 \cos (2 \theta)-\cos (3 \theta) .
\end{aligned}
$$

It can be shown that $u(\theta)>0$ on $(0, \pi)$,

$$
f(\theta)=\frac{v(\theta)}{u(\theta)}=\frac{40-15 \cos (\theta)-24 \cos (2 \theta)-\cos (3 \theta)}{1208+1191 \cos (\theta)+120 \cos (2 \theta)+\cos (3 \theta)}
$$

is monotone increasing on $(0, \pi)$, and $f^{\prime}(\theta)=0$ only for $\theta=0, \pi$. Suppose we want to approximate the eigenvalues of $X_{n}=T_{n}(u)^{-1} T_{n}(v)$ for $n=5000$. Let $\tilde{\lambda}_{j}^{(m)}\left(X_{n}\right)$ be the approximation of $\lambda_{j}\left(X_{n}\right)$ obtained by applying Algorithm 1 with $n_{1}=50 \cdot 2^{m-1}$ and $\alpha=4$. The graph of the errors $\varepsilon_{j, n}^{(m)}$ versus $\theta_{j, n}$ is shown in Fig. 8 for $j=1, \ldots, n$ and $m=1,2,3,4$. The CPU times are reported in Table 2 . 

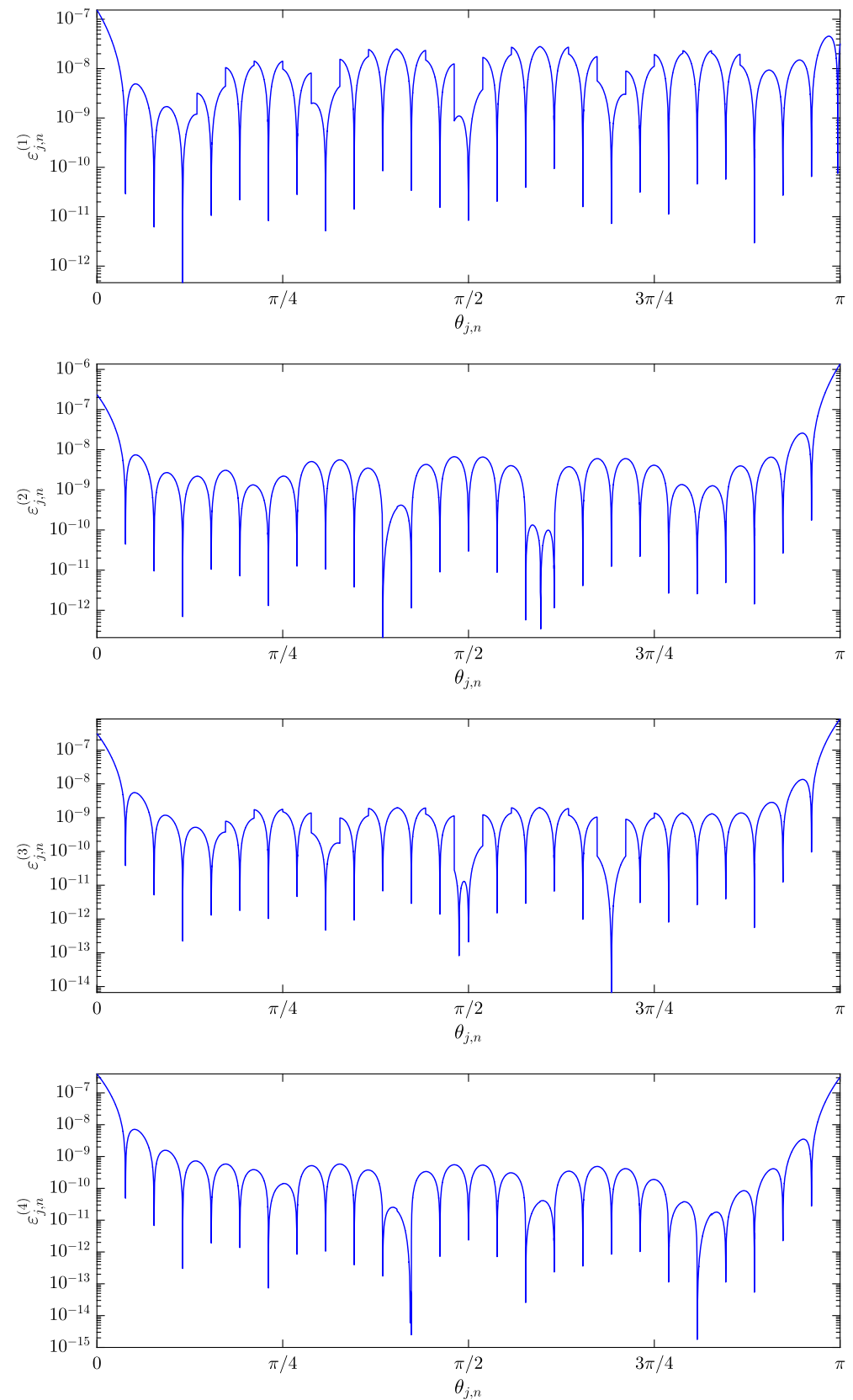

Fig. 6 Example 5: errors $\varepsilon_{j, n}^{(m)}$ versus $\theta_{j, n}$ for $j=1, \ldots, n$, in the case where $u(\theta)=1, v(\theta)=\frac{301}{400}-$ $\cos (\theta)+\frac{1}{5} \cos (2 \theta)+\frac{1}{10} \cos (3 \theta)-\frac{1}{20} \cos (4 \theta)+\frac{1}{400} \cos (6 \theta), n=10000, n_{1}=25$, and $\alpha=4+m$ 

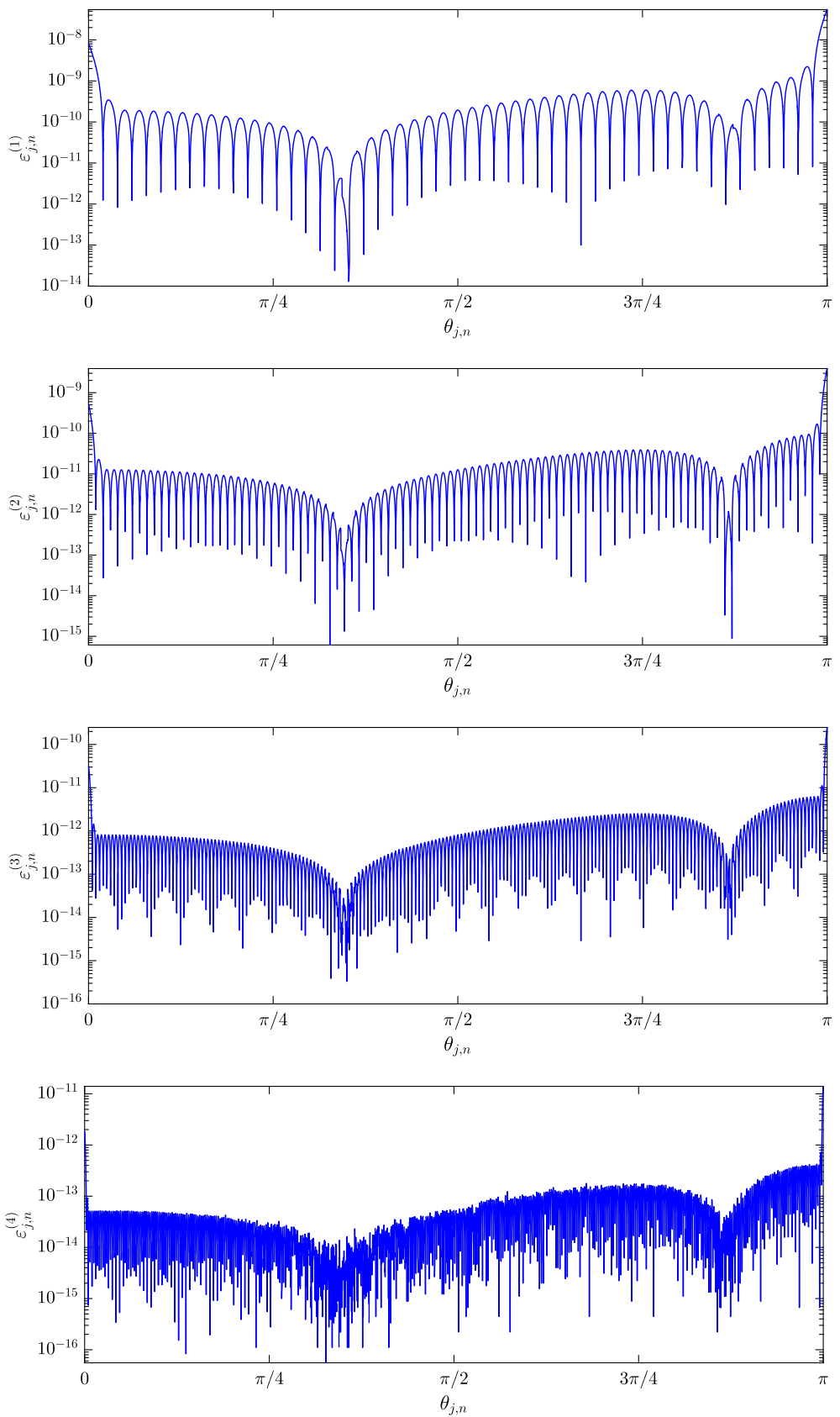

Fig. 7 Example 6: errors $\varepsilon_{j, n}^{(m)}$ versus $\theta_{j, n}$ for $j=1, \ldots, n$, in the case where $u(\theta)=3+2 \cos (\theta)$, $v(\theta)=2-\cos (\theta)-\cos (2 \theta), n=5000, n_{1}=50 \cdot 2^{m-1}$, and $\alpha=4$ 
Table 1 Example 6 (Fig. 7): CPU times for computing the eigenvalues of $X_{n}$ in the case where $u(\theta)=3+2 \cos (\theta)$, $v(\theta)=2-\cos (\theta)-\cos (2 \theta)$, and $n=5000$
Method

CPU time

Algorithm 1 with $n_{1}=50$ and $\alpha=4$

$1.81 \mathrm{~s}$

Algorithm 1 with $n_{1}=100$ and $\alpha=4$

$7.14 \mathrm{~s}$

Algorithm 1 with $n_{1}=200$ and $\alpha=4$

$32.45 \mathrm{~s}$

Algorithm 1 with $n_{1}=400$ and $\alpha=4$

$144.08 \mathrm{~s}$

MATLAB's eig function

Example 8 Let

$$
\begin{aligned}
& u(\theta)=8-3 \cos (\theta)-4 \cos (2 \theta)-\cos (3 \theta), \\
& v(\theta)=\frac{35}{2}-12 \cos (\theta)-6 \cos (2 \theta)+\frac{1}{2} \cos (4 \theta) .
\end{aligned}
$$

It can be shown that $u(\theta)>0$ on $(0, \pi)$,

$$
f(\theta)=\frac{v(\theta)}{u(\theta)}=2-\cos (\theta)
$$

is monotone increasing on $(0, \pi)$, and $f^{\prime}(\theta)=0$ only for $\theta=0, \pi$. Suppose we want to approximate the smallest five eigenvalues of $X_{n}=T_{n}(u)^{-1} T_{n}(v)$ for $n=5000$. Let $\tilde{\lambda}_{j}\left(X_{n}\right)$ be the approximations of $\lambda_{j}\left(X_{n}\right)$ obtained by applying Algorithm 1 with $n_{1}=100$ and $\alpha=4$. Table 3 shows the errors $\varepsilon_{j, n}$ for $j=1, \ldots, 5$, whereas Table 4 compares the CPU times for computing the eigenvalues of $X_{n}$ by using Algorithm 1, MATLAB's eig function, and MATLAB's eigs function (applied to the generalized eigenvalue problem $T_{n}(v) \mathbf{x}=\lambda T_{n}(u) \mathbf{x}$ with $T_{n}(v)$ and $T_{n}(u)$ allocated as sparse matrices through MATLAB's sparse command).

\section{Generalization to the non-monotone case}

With reference to Conjecture 1 , suppose that the function $f=v / u$ is monotone decreasing on $(0, \pi)$. Then, $-f=-v / u$ is monotone increasing on $(0, \pi)$ and, moreover, $T_{n}(u)^{-1} T_{n}(v)=-T_{n}(u)^{-1} T_{n}(-v)$. This immediately implies that Algorithm 1 allows one to compute the eigenvalues of $T_{n}(u)^{-1} T_{n}(v)$ even in the case where $f=v / u$ is monotone decreasing on $(0, \pi)$ : it suffices to apply the algorithm with $X_{n}=T_{n}(u)^{-1} T_{n}(-v)$. Some limitations on the applicability of Algorithm 1 arise when $f$ is non-monotone on $(0, \pi)$. This is precisely the case we are going to investigate in this section. We begin by formulating the following conjecture.

Conjecture 2 Let $u, v$ be RCTPs, with $u>0$ on $(0, \pi)$, and suppose that $f=v / u$ restricted to the interval $I \subseteq(0, \pi)$ is monotone and $f^{-1}(f(I))=I$. Set $X_{n}=$ $T_{n}(u)^{-1} T_{n}(v)$ for all $n$. Then, for every integer $\alpha \geq 0$, every $n$ and every $j=1, \ldots, n$ such that $\theta_{j, n} \in I$, the following asymptotic expansion holds:

$$
\lambda_{\rho_{n}(j)}\left(X_{n}\right)=f\left(\theta_{j, n}\right)+\sum_{k=1}^{\alpha} c_{k}\left(\theta_{j, n}\right) h^{k}+E_{j, n, \alpha},
$$



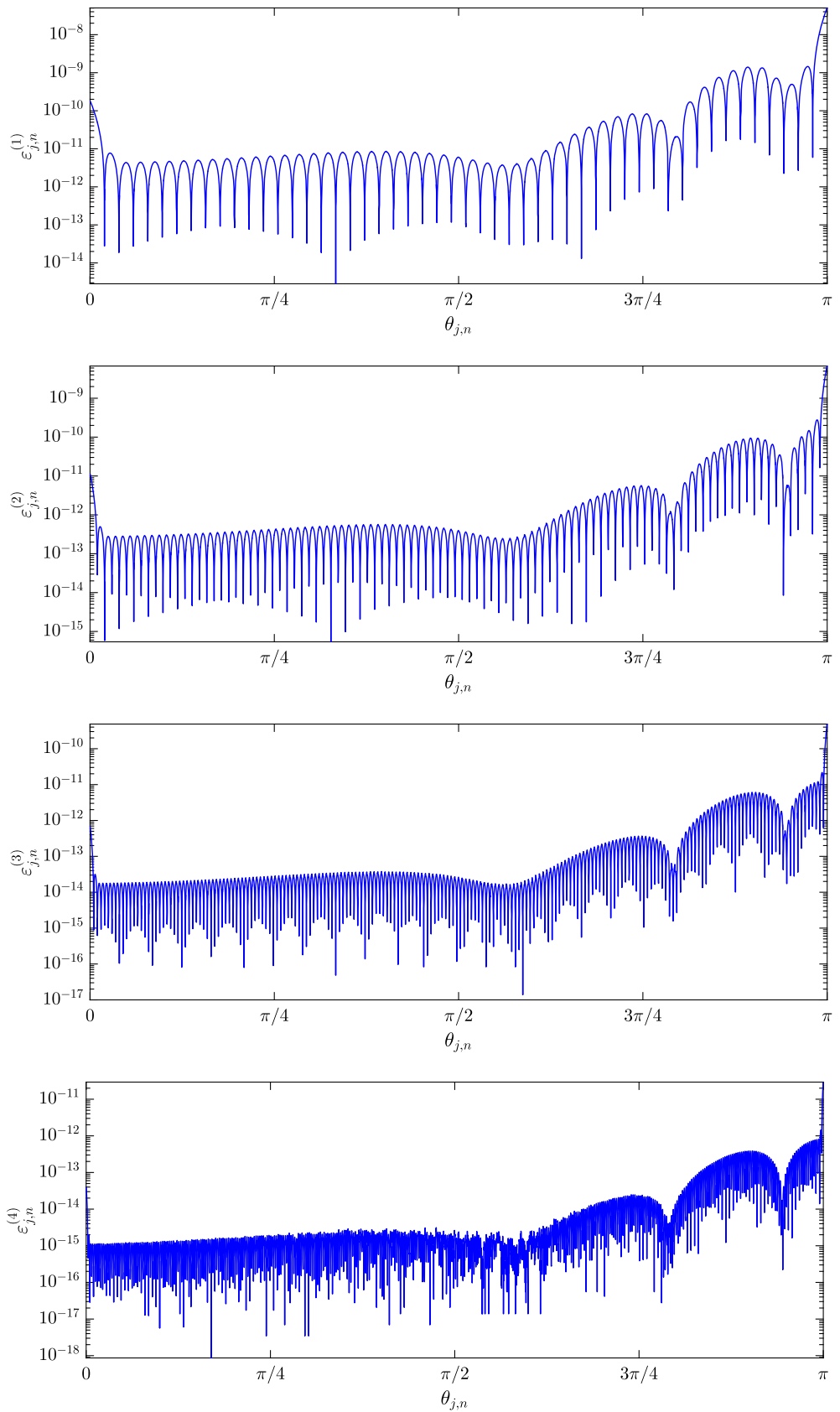

Fig. 8 Example 7: errors $\varepsilon_{j, n}^{(m)}$ versus $\theta_{j, n}$ for $j=1, \ldots, n$, in the case where $u(\theta)=1208+1191 \cos (\theta)+$ $120 \cos (2 \theta)+\cos (3 \theta), v(\theta)=40-15 \cos (\theta)-24 \cos (2 \theta)-\cos (3 \theta), n=5000, n_{1}=50 \cdot 2^{m-1}$, and $\alpha=4$ 
Table 2 Example 7 (Fig. 8): CPU times for computing the eigenvalues of $X_{n}$ in the case where

$u(\theta)=1208+1191 \cos (\theta)+$ $120 \cos (2 \theta)+\cos (3 \theta)$, $v(\theta)=40-15 \cos (\theta)-$ $24 \cos (2 \theta)-\cos (3 \theta)$, and $n=5000$
Method

CPU time

Algorithm 1 with $n_{1}=50$ and $\alpha=4$

$1.69 \mathrm{~s}$

Algorithm 1 with $n_{1}=100$ and $\alpha=4$

$2.77 \mathrm{~s}$

Algorithm 1 with $n_{1}=200$ and $\alpha=4$

$18.30 \mathrm{~s}$

Algorithm 1 with $n_{1}=400$ and $\alpha=4$

$280.27 \mathrm{~s}$

MATLAB's eig function

$1265.55 \mathrm{~s}$

where:

- The eigenvalues of $X_{n}$ are arranged in non-decreasing order, $\lambda_{1}\left(X_{n}\right) \leq \ldots \leq$ $\lambda_{n}\left(X_{n}\right)$.

- $\rho_{n}=\sigma_{n}^{-1}$ is the inverse of $\sigma_{n}$, where $\sigma_{n}$ is a permutation of $\{1, \ldots, n\}$ such that $f\left(\theta_{\sigma_{n}(1), n}\right) \leq \ldots \leq f\left(\theta_{\sigma_{n}(n), n}\right)$.

- $\left\{c_{k}\right\}_{k=1,2, \ldots}$ is a sequence of functions from $I$ to $\mathbb{R}$ which depends only on $u, v$.

- $h=\frac{1}{n+1}$ and $\theta_{j, n}=\frac{j \pi}{n+1}=j \pi h$.

- $E_{j, n, \alpha}=O\left(h^{\alpha+1}\right)$ is the error, which satisfies the inequality $\left|E_{j, n, \alpha}\right| \leq C_{\alpha} h^{\alpha+1}$ for some constant $C_{\alpha}$ depending only on $\alpha, u, v$.

Conjecture 2 is clearly an extension of Conjecture 1 . Indeed, in the case where $f$ is monotone increasing on $(0, \pi)$, if we take $I=(0, \pi)$ and we note that both $\sigma_{n}$ and $\rho_{n}$ reduce to the identity on $\{1, \ldots, n\}$, we see that Conjecture 2 reduces to Conjecture 1 . Conjecture 2 is based on the numerical experiments carried out in $[1,13]$. In the case where $u=1$ identically, it was already formulated in [13]. In the case where $u=1$ identically and $\alpha=0$, it can be formally proved by adapting the argument used by Bogoya, Böttcher, Grudsky, and Maximenko in the proof of [7, Theorem 1.6].

In the situation described in Conjecture 2, we propose the following natural modification of Algorithm 1 for computing the eigenvalues of $X_{n}$ corresponding to the the interval $I$ (that is, the eigenvalues $\lambda_{\rho_{n}(j)}\left(X_{n}\right)$ corresponding to points $\left.\theta_{j, n} \in I\right)$. In what follows, for any integer $n_{1}$, we denote by $n_{1}(I)$ the cardinality of $\left\{\theta_{1, n_{1}}, \ldots, \theta_{n_{1}, n_{1}}\right\} \cap I$.

Algorithm 2 With the notation introduced in Conjecture 2, given two RCTPs $u, v$ (with $u>0$ on $(0, \pi)$ and $f=v / u$ such that $f$ restricted to the interval $I \subseteq(0, \pi)$ is monotone and $\left.f^{-1}(f(I))=I\right)$, three integers $n, n_{1}, \alpha \in \mathbb{N}$ with $n_{1}(I) \geq \alpha$ and

Table 3 Example 8: errors $\varepsilon_{j, n}$ for $j=1, \ldots, 5$, in the case where $u(\theta)=8-3 \cos (\theta)-4 \cos (2 \theta)-$ $\cos (3 \theta), v(\theta)=\frac{35}{2}-12 \cos (\theta)-6 \cos (2 \theta)+\frac{1}{2} \cos (4 \theta), n=5000, n_{1}=100$, and $\alpha=4$

\begin{tabular}{llllll}
\hline$j$ & 1 & 2 & 3 & 4 & 5 \\
$\varepsilon_{j, n}$ & $1.56 \cdot 10^{-6}$ & $1.42 \cdot 10^{-6}$ & $1.47 \cdot 10^{-6}$ & $1.34 \cdot 10^{-6}$ & $1.39 \cdot 10^{-6}$ \\
\hline
\end{tabular}


Table 4 Example 8: CPU times for computing the smallest five eigenvalues of $X_{n}$ in the case where $u(\theta)=8-3 \cos (\theta)-$ $4 \cos (2 \theta)-\cos (3 \theta)$, $v(\theta)=\frac{35}{2}-12 \cos (\theta)-$ $6 \cos (2 \theta)+\frac{1}{2} \cos (4 \theta)$, and $n=5000$

\section{Method}

CPU time

Algorithm 1 with $n_{1}=100$ and $\alpha=4$

$1.13 \mathrm{~s}$

MATLAB's eig function

$346.21 \mathrm{~s}$

MATLAB's eigs function

$S \subseteq I$, we compute approximations of the eigenvalues $\left\{\lambda_{\rho_{n}(j)}\left(X_{n}\right): \theta_{j, n} \in S\right\}$ as follows:

1. For $j_{1}=1, \ldots, n_{1}$ such that $\theta_{j_{1}, n_{1}} \in I$ compute $\tilde{c}_{1}\left(\theta_{j_{1}, n_{1}}\right), \ldots, \tilde{c}_{\alpha}\left(\theta_{j_{1}, n_{1}}\right)$ by solving the linear system

$$
\left\{\begin{array}{l}
E_{j_{1}, n_{1}, 0}=\tilde{c}_{1}\left(\theta_{j_{1}, n_{1}}\right) h_{1}+\tilde{c}_{2}\left(\theta_{j_{1}, n_{1}}\right) h_{1}^{2}+\ldots+\tilde{c}_{\alpha}\left(\theta_{j_{1}, n_{1}}\right) h_{1}^{\alpha} \\
E_{j_{2}, n_{2}, 0}=\tilde{c}_{1}\left(\theta_{j_{1}, n_{1}}\right) h_{2}+\tilde{c}_{2}\left(\theta_{j_{1}, n_{1}}\right) h_{2}^{2}+\ldots+\tilde{c}_{\alpha}\left(\theta_{j_{1}, n_{1}}\right) h_{2}^{\alpha} \\
\vdots \\
E_{j_{\alpha}, n_{\alpha}, 0}=\tilde{c}_{1}\left(\theta_{j_{1}, n_{1}}\right) h_{\alpha}+\tilde{c}_{2}\left(\theta_{j_{1}, n_{1}}\right) h_{\alpha}^{2}+\ldots+\tilde{c}_{\alpha}\left(\theta_{j_{1}, n_{1}}\right) h_{\alpha}^{\alpha}
\end{array}\right.
$$

where $n_{k}=2^{k-1}\left(n_{1}+1\right)-1, j_{k}=2^{k-1} j_{1}$, and

$$
E_{j_{k}, n_{k}, 0}=\lambda_{\rho_{n_{k}}\left(j_{k}\right)}\left(X_{n_{k}}\right)-f\left(\theta_{j_{1}, n_{1}}\right), \quad k=1, \ldots, \alpha .
$$

2. For $j=1, \ldots, n$ such that $\theta_{j, n} \in S$

- For $k=1, \ldots, \alpha$

- Determine $\alpha-k+1$ points $\theta^{(1)}, \ldots, \theta^{(\alpha-k+1)} \in\left\{\theta_{1, n_{1}}, \ldots, \theta_{n_{1}, n_{1}}\right\} \cap I$ which are closest to $\theta_{j, n}$

- Compute $\tilde{c}_{k, j}\left(\theta_{j, n}\right)$, where $\tilde{c}_{k, j}(\theta)$ is the interpolation polynomial of $\left(\theta^{(1)}, \tilde{c}_{k}\left(\theta^{(1)}\right)\right), \ldots,\left(\theta^{(\alpha-k+1)}, \tilde{c}_{k}\left(\theta^{(\alpha-k+1)}\right)\right)$

- Compute $\tilde{\lambda}_{\rho_{n}(j)}\left(X_{n}\right)=f\left(\theta_{j, n}\right)+\sum_{k=1}^{\alpha} \tilde{c}_{k, j}\left(\theta_{j, n}\right) h^{k}$

3. Return $\left\{\tilde{\lambda}_{\rho_{n}(j)}\left(X_{n}\right): \theta_{j, n} \in S\right\}$ as an approximation to $\left\{\lambda_{\rho_{n}(j)}\left(X_{n}\right): \theta_{j, n} \in S\right\}$

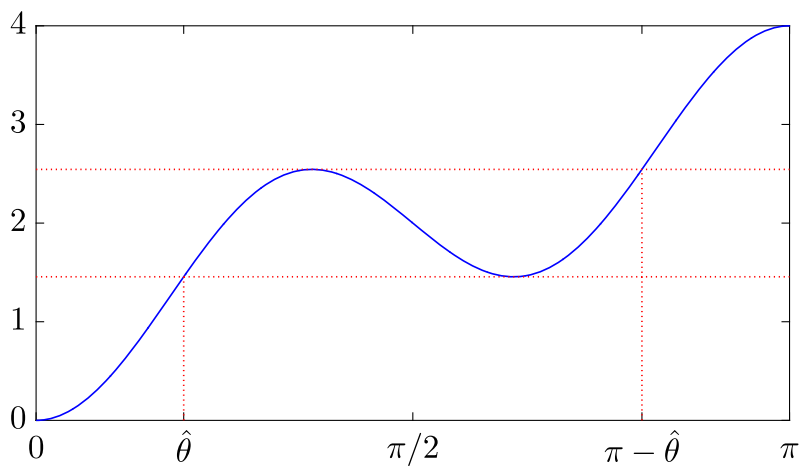

Fig. 9 Example 9: graph of $f(\theta)=v(\theta) / u(\theta)=2-\cos (\theta)-\cos (3 \theta)$ over $(0, \pi)$ 

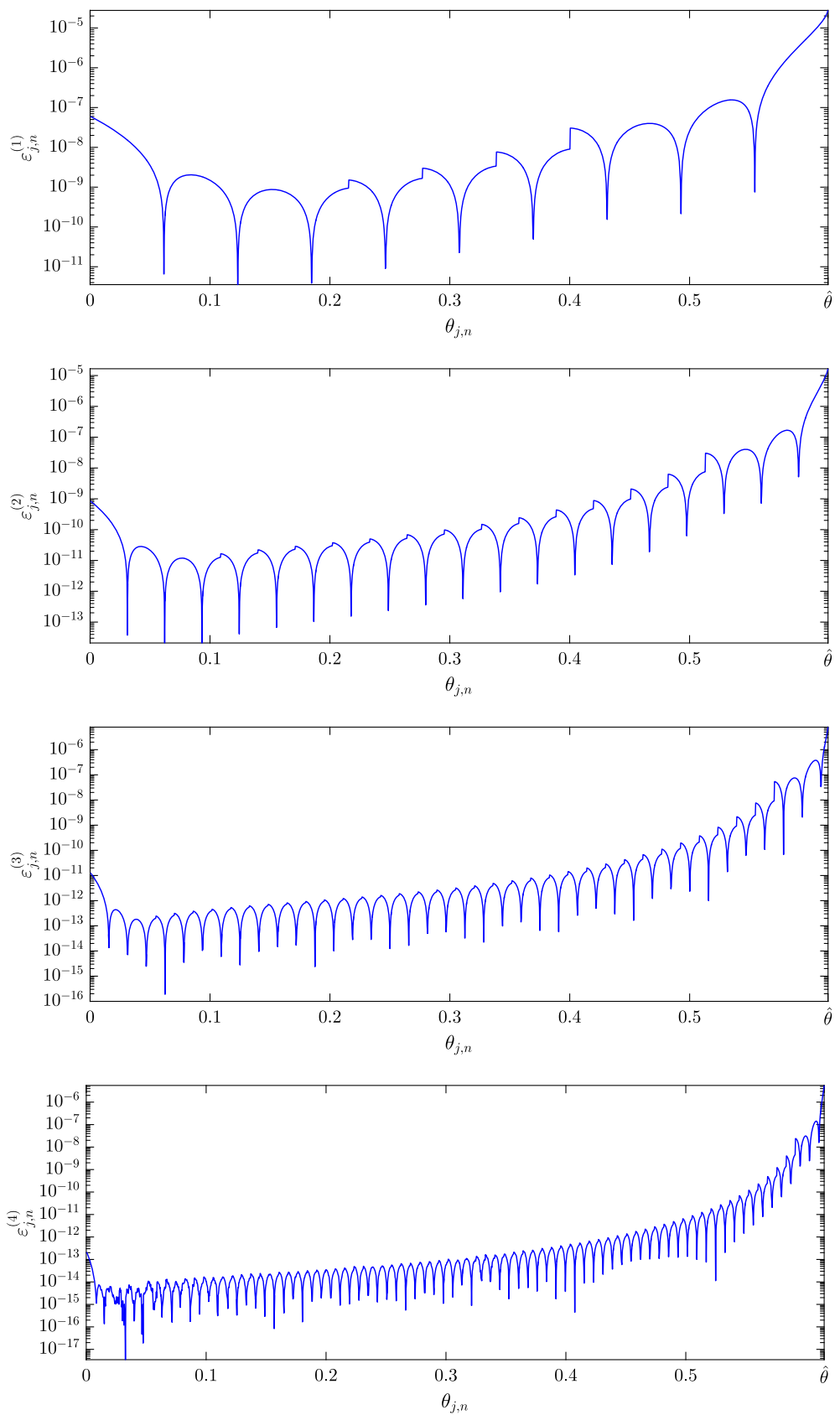

Fig. 10 Example 9: errors $\varepsilon_{j, n}^{(m)}$ versus $\theta_{j, n}$ for $\theta_{j, n} \in I=(0, \hat{\theta})$, in the case where $u(\theta)=1, v(\theta)=$ $2-\cos (\theta)-\cos (3 \theta), n=10000, n_{1}=50 \cdot 2^{m-1}$, and $\alpha=5$ 


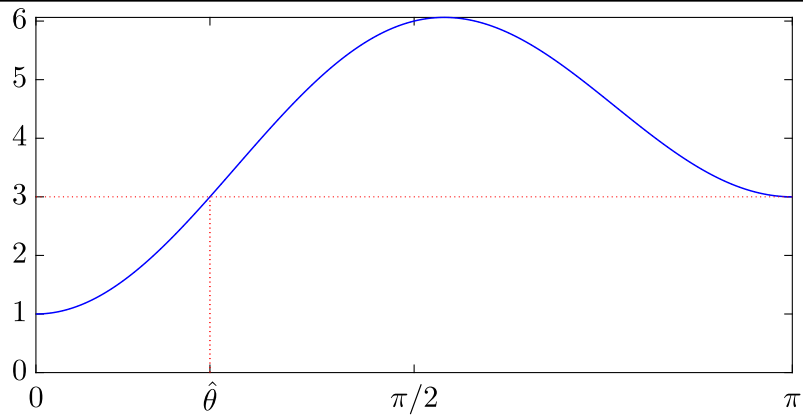

Fig. 11 Example 10: graph of $f(\theta)=v(\theta) / u(\theta)=4-\cos (\theta)-2 \cos (2 \theta)$ over $(0, \pi)$

Example 9 Let

$$
\begin{aligned}
& u(\theta)=1, \\
& v(\theta)=2-\cos (\theta)-\cos (3 \theta) .
\end{aligned}
$$

The graph of $f(\theta)=v(\theta) / u(\theta)=v(\theta)$ is depicted in Fig. 9. The hypotheses of Conjecture 2 are satisfied with either $I=(0, \hat{\theta})$ or $I=(\pi-\hat{\theta}, \pi)$, where $\hat{\theta}=$ $0.61547970867038 \ldots$ To fix the ideas, let $I=(0, \hat{\theta})$. Note that any permutation $\sigma_{n}$ which sorts the samples $f\left(\theta_{1, n}\right), \ldots, f\left(\theta_{n, n}\right)$ in non-decreasing order is such that $\sigma_{n}(j)=j$ whenever $\theta_{j, n} \in I$. As a consequence, $\rho_{n}(j)=j$ whenever $\theta_{j, n} \in I$. Set $X_{n}=T_{n}(u)^{-1} T_{n}(v)=T_{n}(f)$ and let $\left\{\tilde{\lambda}_{j}^{(m)}\left(X_{n}\right): \theta_{j, n} \in I\right\}$ be the approximation of $\left\{\lambda_{j}\left(X_{n}\right): \theta_{j, n} \in I\right\}$ obtained for $n=10000$ by applying Algorithm 2 with $n_{1}=$ $50 \cdot 2^{m-1}, \alpha=5$, and $S=I$. The graph of the errors $\varepsilon_{j, n}^{(m)}=\left|\lambda_{j}\left(X_{n}\right)-\tilde{\lambda}_{j}^{(m)}\left(X_{n}\right)\right|$ versus $\theta_{j, n}$ is shown in Fig. 10 for $\theta_{j, n} \in I$ and $m=1,2,3,4$. We note that the error $\varepsilon_{j, n}^{(m)}$ tends to increase as $\theta_{j, n}$ moves toward $\hat{\theta}$, that is, as $\theta_{j, n}$ approaches to exit the interval $I$ over which $f$ satisfies the assumptions of Conjecture 2. Moreover, in a neighborhood of $\hat{\theta}$, the error decreases very slowly. This phenomenon is related to the fact that the expansion (7) does not hold in $[\hat{\theta}, \pi-\hat{\theta}]$ and, in fact, the errors $E_{j, n, 0}=$ $\lambda_{\rho_{n}(j)}\left(X_{n}\right)-f\left(\theta_{j, n}\right)$ have a wild behavior inside this interval; see [13, Fig. 7].

\section{Example 10 Let}

$$
\begin{aligned}
& u(\theta)=2+\cos (3 \theta), \\
& v(\theta)=8-3 \cos (\theta)-\frac{9}{2} \cos (2 \theta)+4 \cos (3 \theta)-\frac{1}{2} \cos (4 \theta)-\cos (5 \theta) .
\end{aligned}
$$

The graph of $f(\theta)=v(\theta) / u(\theta)=4-\cos (\theta)-2 \cos (2 \theta)$ is depicted in Fig. 11. The hypotheses of Conjecture 2 are satisfied with $I=(0, \hat{\theta})$, where $\hat{\theta}=0.72273424781341 \ldots$ Any permutation $\sigma_{n}$ which sorts the samples $f\left(\theta_{1, n}\right), \ldots, f\left(\theta_{n, n}\right)$ in non-decreasing order is such that $\sigma_{n}(j)=j$ whenever $\theta_{j, n} \in I$. As a consequence, $\rho_{n}(j)=j$ whenever $\theta_{j, n} \in I$. Set $X_{n}=T_{n}(u)^{-1} T_{n}(v)$ and let $\left\{\tilde{\lambda}_{j}^{(m)}\left(X_{n}\right): \theta_{j, n} \in I\right\}$ be the approximation of $\left\{\lambda_{j}\left(X_{n}\right): \theta_{j, n} \in I\right\}$ obtained for $n=5000$ by applying Algorithm 2 with $n_{1}=25 \cdot 2^{m-1}, \alpha=5$, and $S=I$. The graph of the errors $\varepsilon_{j, n}^{(m)}=\left|\lambda_{j}\left(X_{n}\right)-\tilde{\lambda}_{j}^{(m)}\left(X_{n}\right)\right|$ versus $\theta_{j, n}$ is shown in Fig. 12 for 

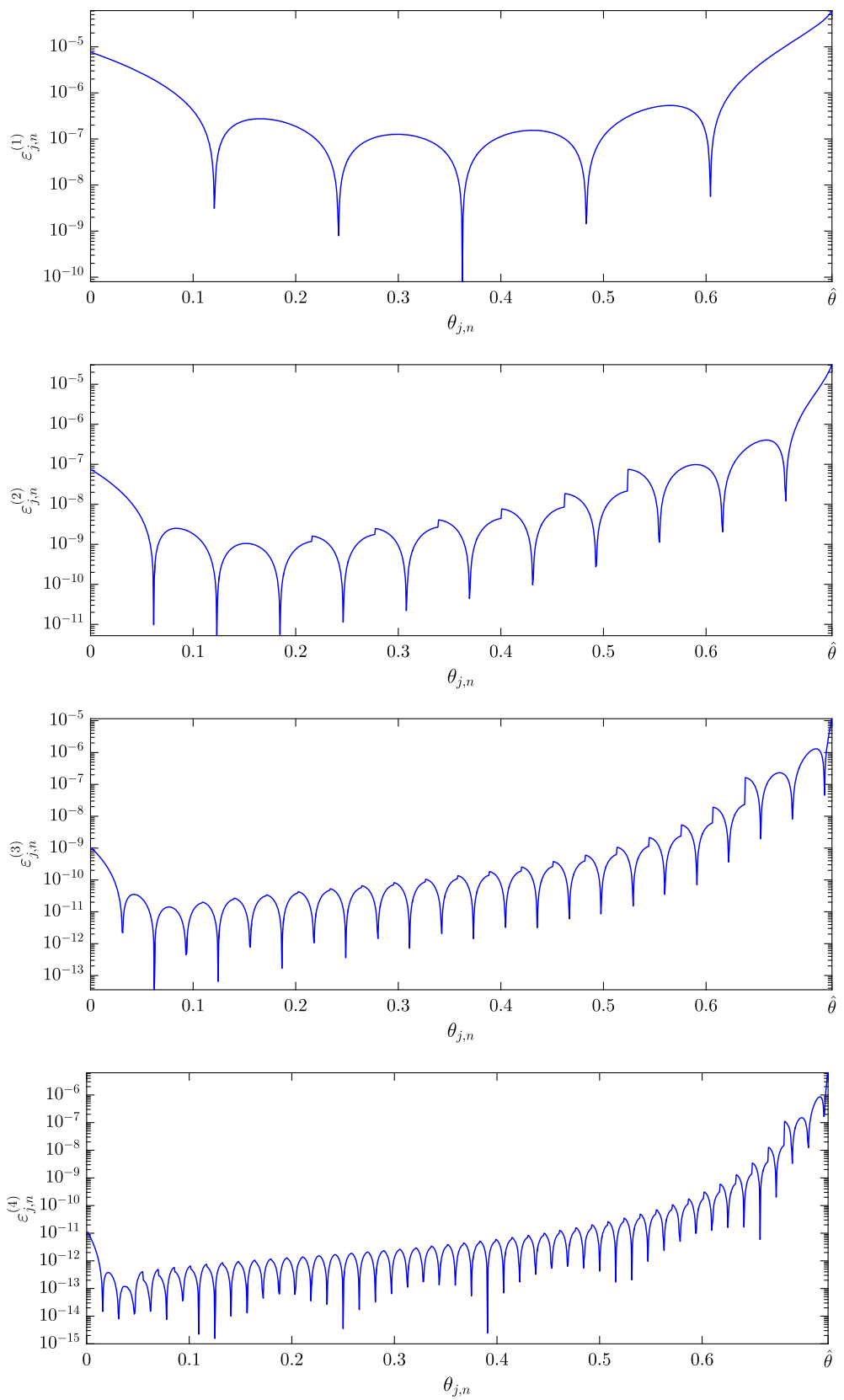

Fig. 12 Example 10: errors $\varepsilon_{j, n}^{(m)}$ versus $\theta_{j, n}$ for $\theta_{j, n} \in I=(0, \hat{\theta})$, in the case where $u(\theta)=2+\cos (3 \theta)$, $v(\theta)=8-3 \cos (\theta)-\frac{9}{2} \cos (2 \theta)+4 \cos (3 \theta)-\frac{1}{2} \cos (4 \theta)-\cos (5 \theta), n=5000, n_{1}=25 \cdot 2^{m-1}$, and $\alpha=5$

$\theta_{j, n} \in I$ and $m=1,2,3,4$. Considerations analogous to those in Example 10 apply also in this case. 


\section{Conclusions and perspectives}

We have proposed and analyzed a matrix-less parallel interpolation-extrapolation algorithm for computing the eigenvalues of preconditioned banded symmetric Toeplitz matrices of the form $T_{n}(u)^{-1} T_{n}(v)$, where $u, v$ are RCTPs, $u>0$ on $(0, \pi)$, and $f=v / u$ is monotone on $(0, \pi)$. We have illustrated the performance of the algorithm through numerical experiments, and we have presented its generalization to the case where $f=v / u$ is non-monotone. We conclude by suggesting two possible future lines of research:

- Algorithm 1, as well as its generalized version for the non-monotone case (Algorithm 2), is based on a local interpolation strategy, as described in Section 2.1. An interesting topic for future research could be the following: try another kind of approximation (for example, an higher-order spline approximation) to see whether this reduces the errors and accelerates the convergence of both these algorithms.

- Understand whether an asymptotic eigenvalue expansion analogous to (7) holds without the hypothesis that $f$ restricted to some interval $I \subseteq(0, \pi)$ is monotone and satisfies $f^{-1}(f(I))=I$. Such a result would eliminate any limitation in the applicability of Algorithm 2 (provided that the latter is properly modified according to the new expansion).

Funding Information The research of Sven-Erik Ekström is cofinanced by the Graduate School in Mathematics and Computing (FMB) and Uppsala University. Carlo Garoni is a Marie-Curie fellow of the Italian INdAM (Istituto Nazionale di Alta Matematica) under grant agreement PCOFUND-GA-2012600198.

Open Access This article is distributed under the terms of the Creative Commons Attribution 4.0 International License (http://creativecommons.org/licenses/by/4.0/), which permits unrestricted use, distribution, and reproduction in any medium, provided you give appropriate credit to the original author(s) and the source, provide a link to the Creative Commons license, and indicate if changes were made.

\section{Appendix A}

This appendix collects the proofs of Theorems 1 and 2.

Proof of Theorem 1 We follow the argument in [1, Section 2]. Equations (2) and (4) can be rewritten as

$$
\begin{aligned}
& A\left(h_{1}, \ldots, h_{1}\right) \mathbf{c}\left(j_{1}\right)=\mathbf{E}_{0}\left(j_{1}\right)-\mathbf{E}_{\alpha}\left(j_{1}\right) \\
& A\left(h_{1}, \ldots, h_{1}\right) \tilde{\mathbf{c}}\left(j_{1}\right)=\mathbf{E}_{0}\left(j_{1}\right),
\end{aligned}
$$


where

$$
\begin{array}{cc}
\mathbf{c}\left(j_{1}\right)=\left[\begin{array}{c}
c_{1}\left(\theta_{j_{1}, n_{1}}\right) \\
\vdots \\
c_{\alpha}\left(\theta_{j_{1}, n_{1}}\right)
\end{array}\right], & \tilde{\mathbf{c}}\left(j_{1}\right)=\left[\begin{array}{c}
\tilde{c}_{1}\left(\theta_{j_{1}, n_{1}}\right) \\
\vdots \\
\tilde{c}_{\alpha}\left(\theta_{j_{1}, n_{1}}\right)
\end{array}\right], \\
\mathbf{E}_{0}\left(j_{1}\right)=\left[\begin{array}{c}
E_{j_{1}, n_{1}, 0} \\
\vdots \\
E_{j_{\alpha}, n_{\alpha}, 0}
\end{array}\right], & \mathbf{E}_{\alpha}\left(j_{1}\right)=\left[\begin{array}{c}
E_{j_{1}, n_{1}, \alpha} \\
\vdots \\
E_{j_{\alpha}, n_{\alpha}, \alpha}
\end{array}\right],
\end{array}
$$

and

$$
A\left(h_{1}, \ldots, h_{\alpha}\right)=\operatorname{diag}\left(h_{1}, \ldots, h_{\alpha}\right) V\left(h_{1}, \ldots, h_{\alpha}\right),
$$

with $V\left(h_{1}, \ldots, h_{\alpha}\right)$ being the Vandermonde matrix associated with the nodes $h_{1}, \ldots, h_{\alpha}$,

$$
V\left(h_{1}, \ldots, h_{\alpha}\right)=\left[\begin{array}{ccccc}
1 & h_{1} & h_{1}^{2} & \cdots & h_{1}^{\alpha-1} \\
1 & h_{2} & h_{2}^{2} & \cdots & h_{2}^{\alpha-1} \\
\vdots & \vdots & \vdots & & \vdots \\
1 & h_{\alpha} & h_{\alpha}^{2} & \cdots & h_{\alpha}^{\alpha-1}
\end{array}\right]
$$

By (9), (10), and (12), we have

$$
\tilde{\mathbf{c}}\left(j_{1}\right)-\mathbf{c}\left(j_{1}\right)=A\left(h_{1}, \ldots, h_{\alpha}\right)^{-1} \mathbf{E}_{\alpha}\left(j_{1}\right)=V\left(h_{1}, \ldots, h_{\alpha}\right)^{-1} \mathbf{F}_{\alpha}\left(j_{1}\right),
$$

where

$$
\mathbf{F}_{\alpha}\left(j_{1}\right)=\operatorname{diag}\left(h_{1}, \ldots, h_{\alpha}\right)^{-1} \mathbf{E}_{\alpha}\left(j_{1}\right)=\left[\begin{array}{c}
E_{j_{1}, n_{1}, \alpha} / h_{1} \\
\vdots \\
E_{j_{\alpha}, n_{\alpha}, \alpha} / h_{\alpha}
\end{array}\right] .
$$

Note that, by (3),

$$
\left|\left(\mathbf{F}_{\alpha}\left(j_{1}\right)\right)_{k}\right|=\left|E_{j_{k}, n_{k}, \alpha} / h_{k}\right| \leq C_{\alpha} h_{k}^{\alpha}, \quad k=1, \ldots, \alpha .
$$

The inverse of $V\left(h_{1}, \ldots, h_{\alpha}\right)$ is explicitly given by

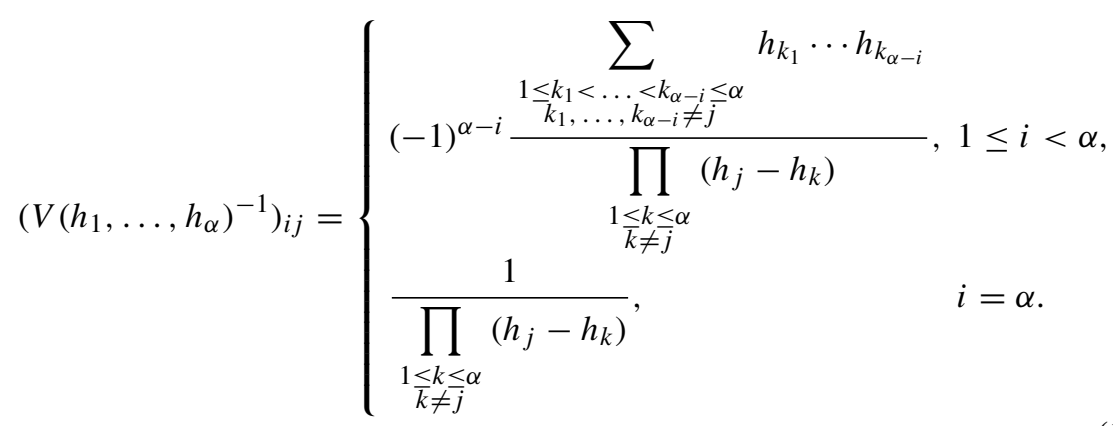


Taking into account (13) and the equation $h_{k}=2^{1-k} h_{1}$ for $k=1, \ldots, \alpha$, we obtain the following:

- $\quad$ For $i=\alpha$,

$$
\begin{aligned}
& \left|\tilde{c}_{\alpha}\left(\theta_{j_{1}, n_{1}}\right)-c_{\alpha}\left(\theta_{j_{1}, n_{1}}\right)\right|=\left|\left(\tilde{\mathbf{c}}\left(j_{1}\right)-\mathbf{c}\left(j_{1}\right)\right)_{\alpha}\right| \\
= & \left|\sum_{j=1}^{\alpha}\left(V\left(h_{1}, \ldots, h_{\alpha}\right)^{-1}\right)_{\alpha j}\left(\mathbf{F}_{\alpha}\left(j_{1}\right)\right)_{j}\right| \\
\leq & \sum_{j=1}^{\alpha} \frac{\left|\left(\mathbf{F}_{\alpha}\left(j_{1}\right)\right)_{j}\right|}{\prod_{\substack{1 \leq k \leq \alpha \\
k \neq j}}\left|h_{j}-h_{k}\right|} \leq \sum_{j=1}^{\alpha} \frac{C_{\alpha} h_{j}^{\alpha}}{h_{j}^{\alpha-1} \prod_{\substack{1 \leq k \leq \alpha \\
k \neq j}}\left|1-h_{k} / h_{j}\right|} \\
= & C_{\alpha} h_{1} \sum_{j=1}^{\alpha} \frac{2^{1-j}}{\prod_{\substack{1 \leq k \leq \alpha \\
k \neq j}}\left|1-2^{j-k}\right|}=A(\alpha) h_{1},
\end{aligned}
$$

with $A(\alpha)$ depending only on $\alpha, u, v$ just like $C_{\alpha}$.

- For $1 \leq i<\alpha$,

$$
\begin{aligned}
& \left|\tilde{c}_{i}\left(\theta_{j_{1}, n_{1}}\right)-c_{i}\left(\theta_{j_{1}, n_{1}}\right)\right|=\left|\left(\tilde{\mathbf{c}}\left(j_{1}\right)-\mathbf{c}\left(j_{1}\right)\right)_{i}\right| \\
& =\left|\sum_{j=1}^{\alpha}\left(V\left(h_{1}, \ldots, h_{\alpha}\right)^{-1}\right)_{i j}\left(\mathbf{F}_{\alpha}\left(j_{1}\right)\right)_{j}\right|
\end{aligned}
$$

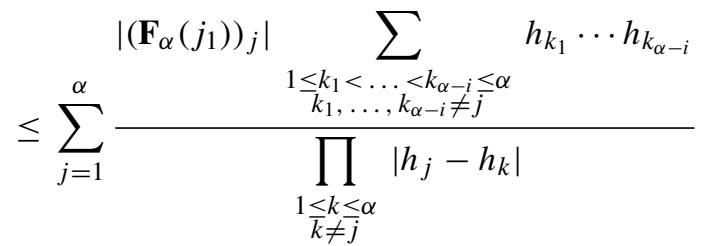

$$
\begin{aligned}
& \leq \sum_{j=1}^{\alpha} \frac{\sum_{\substack{\alpha \\
1 \leq k_{1}<\ldots<k_{\alpha-i} \leq \alpha \\
k_{1}, \ldots, k_{\alpha-i} \neq j}} h_{k_{1}} \cdots h_{k_{\alpha-i}}}{h_{j}^{\alpha-1} \prod_{\substack{1 \leq k \leq \alpha \\
k \neq j}}\left|1-h_{k} / h_{j}\right|} \\
& =C_{\alpha} h_{1}^{\alpha-i+1} \sum_{j=1}^{\alpha} \frac{\sum_{\substack{1-j \\
1 \leq k_{1}<\ldots<k_{\alpha-i} \leq \alpha \\
k_{1}, \ldots, k_{\alpha-i} \neq j}} 2^{1-k_{1}} \cdots 2^{1-k_{\alpha-i}}}{\prod_{\substack{1 \leq k \leq \alpha \\
k \neq j}}\left|1-2^{j-k}\right|} \\
& =A(\alpha, i) h_{1}^{\alpha-i+1},
\end{aligned}
$$

with $A(\alpha, i)$ depending only on $\alpha, i, u, v$. 
In conclusion, Theorem 1 is proved with $A_{\alpha}=\max _{i=1, \ldots, \alpha} A(\alpha, i)$, where $A(\alpha, \alpha)=$ $A(\alpha)$.

Proof of Theorem 2 Let $L_{1}, \ldots, L_{\alpha-k+1}$ be the Lagrange polynomials associated with the nodes $\theta^{(1)}, \ldots, \theta^{(\alpha-k+1)}$,

$$
L_{r}(\theta)=\prod_{\substack{s=1 \\ s \neq r}}^{\alpha-k+1} \frac{\theta-\theta^{(s)}}{\theta^{(r)}-\theta^{(s)}}, \quad r=1, \ldots, \alpha-k+1
$$

The interpolation polynomial of the data $\left(\theta^{(1)}, \tilde{c}_{k}\left(\theta^{(1)}\right)\right), \ldots,\left(\theta^{(\alpha-k+1)}, \tilde{c}_{k}\left(\theta^{(\alpha-k+1)}\right)\right)$ is

$$
\tilde{c}_{k, j}(\theta)=\sum_{r=1}^{\alpha-k+1} \tilde{c}_{k}\left(\theta^{(r)}\right) L_{r}(\theta)
$$

and the interpolation polynomial of the data $\left(\theta^{(1)}, c_{k}\left(\theta^{(1)}\right)\right), \ldots,\left(\theta^{(\alpha-k+1)}\right.$, $\left.c_{k}\left(\theta^{(\alpha-k+1)}\right)\right)$ is

$$
p(\theta)=\sum_{r=1}^{\alpha-k+1} c_{k}\left(\theta^{(r)}\right) L_{r}(\theta)
$$

Considering that $\theta^{(1)}, \ldots, \theta^{(\alpha-k+1)}$ are $\alpha-k+1$ points from $\left\{\theta_{1, n_{1}}, \ldots, \theta_{n_{1}, n_{1}}\right\}$ which are closest to $\theta_{j, n}$, the length of the smallest interval $I$ containing the nodes $\theta^{(1)}, \ldots, \theta^{(\alpha-k+1)}$ and the point $\theta_{j, n}$ is bounded by $(\alpha-k+1) \pi h_{1}$. Hence, by Theorem 1 , for all $\theta \in I$ we have

$$
\begin{aligned}
\left|\tilde{c}_{k, j}(\theta)-p(\theta)\right| & \leq \sum_{r=1}^{\alpha-k+1}\left|\tilde{c}_{k, j}\left(\theta^{(r)}\right)-c_{k}\left(\theta^{(r)}\right)\right| \prod_{\substack{s=1 \\
s \neq r}}^{\alpha-k+1} \frac{\left|\theta-\theta^{(s)}\right|}{\left|\theta^{(r)}-\theta^{(s)}\right|} \\
& \leq \sum_{r=1}^{\alpha-k+1} A_{\alpha} h_{1}^{\alpha-k+1} \prod_{\substack{s=1 \\
s \neq r}}^{\alpha-k+1} \frac{(\alpha-k+1) \pi h_{1}}{\pi h_{1}} \\
& =A_{\alpha} h_{1}^{\alpha-k+1}(\alpha-k+1)^{\alpha-k+1} .
\end{aligned}
$$

Since $c_{k} \in C^{\alpha-k+1}([0, \pi])$ by assumption, from interpolation theory we know that for every $\theta \in I$ there exists $\xi(\theta) \in I$ such that

$$
c_{k}(\theta)-p(\theta)=\frac{c_{k}^{(\alpha-k+1)}(\xi(\theta))}{(\alpha-k+1) !} \prod_{r=1}^{\alpha-k+1}\left(\theta-\theta^{(r)}\right) ;
$$


see, e.g., [12, Theorem 3.1.1]. Thus, for all $\theta \in I$, we have

$$
\begin{aligned}
\left|c_{k}(\theta)-p(\theta)\right| & \leq \frac{\left|c_{k}^{(\alpha-k+1)}(\xi(\theta))\right|}{(\alpha-k+1) !} \prod_{r=1}^{\alpha-k+1}\left|\theta-\theta^{(r)}\right| \\
& \leq \frac{\left\|c_{k}^{(\alpha-k+1)}\right\|_{\infty}}{(\alpha-k+1) !} \prod_{r=1}^{\alpha-k+1}(\alpha-k+1) \pi h_{1} \\
& =\frac{(\alpha-k+1)^{\alpha-k+1} \pi^{\alpha-k+1}\left\|c_{k}^{(\alpha-k+1)}\right\|_{\infty}}{(\alpha-k+1) !} h_{1}^{\alpha-k+1} .
\end{aligned}
$$

From (15) and (16) we obtain

$$
\left|c_{k}(\theta)-\tilde{c}_{k, j}(\theta)\right| \leq B(k, \alpha) h_{1}^{\alpha-k+1} \leq B_{\alpha} h_{1}^{\alpha-k+1}, \quad \theta \in I,
$$

where

$$
B(k, \alpha)=\frac{(\alpha-k+1)^{\alpha-k+1} \pi^{\alpha-k+1}\left\|c_{k}^{(\alpha-k+1)}\right\|_{\infty}}{(\alpha-k+1) !}+A_{\alpha}(\alpha-k+1)^{\alpha-k+1}
$$

and $B_{\alpha}=\max _{i=1, \ldots, \alpha} B(i, \alpha)$. Since $\theta_{j, n} \in I$, it is clear that (6) follows from (17).

\section{Appendix B}

\section{This appendix provides a plain MATLAB implementation of Algorithm 1.}

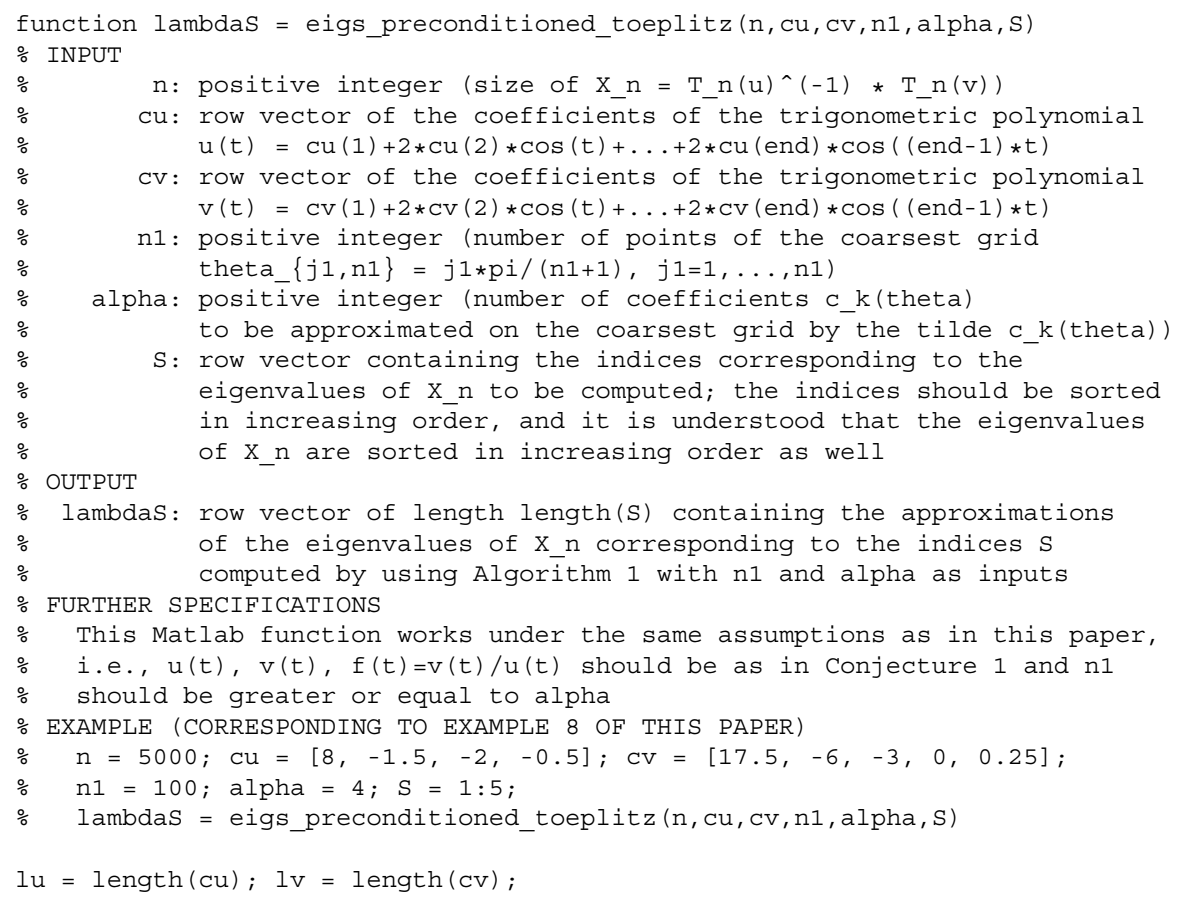




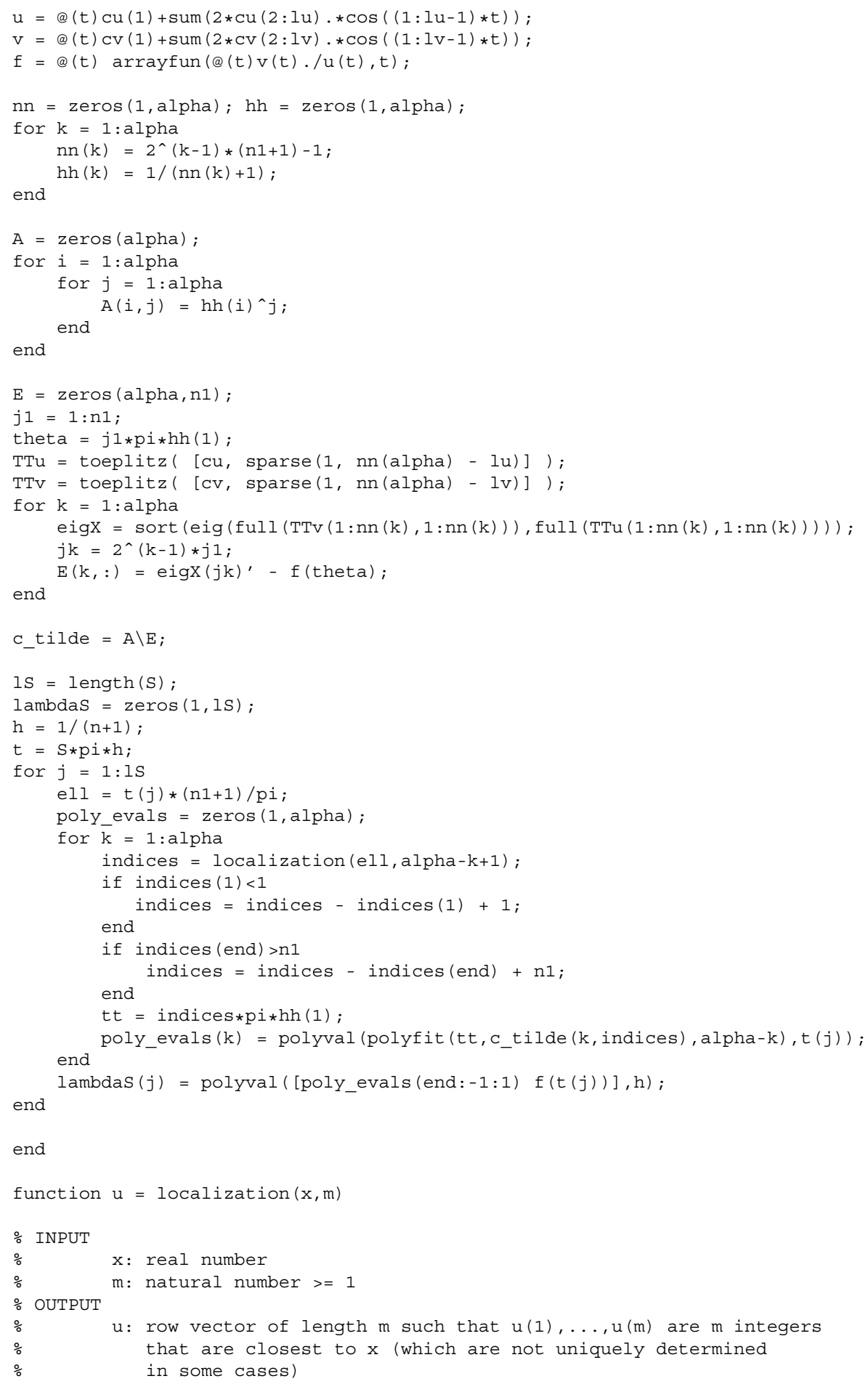

end

function $\mathrm{u}=$ localization $(\mathrm{x}, \mathrm{m})$

$\div$ INPUT

x: real number

$\div \quad m$ : natural number $>=1$

$\%$ OUTPUT

$\% \quad u$ : row vector of length $m$ such that $u(1), \ldots, u(m)$ are $m$ integers

$\div \quad$ that are closest to $\mathrm{x}$ (which are not uniquely determined

in some cases) 


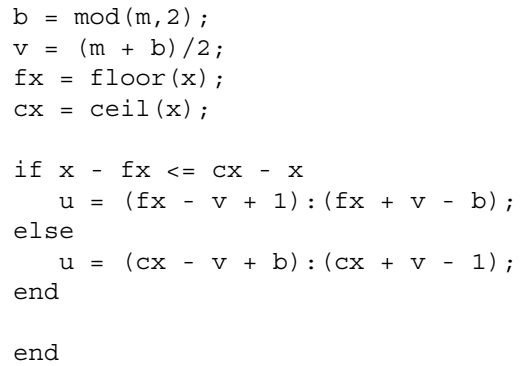

\section{References}

1. Ahmad, F., Al-Aidarous, E.S., Alrehaili, D.A., Ekström, S.-E., Furci, I., Serra-Capizzano, S.: Are the eigenvalues of preconditioned banded symmetric Toeplitz matrices known in almost closed form? Numer. Alg. (in press). https://doi.org/10.1007/s11075-017-0404-z

2. Arbenz, P.: Computing the eigenvalues of banded symmetric Toeplitz matrices. SIAM J. Sci. Stat. Comput. 12, 743-754 (1991)

3. Badía, J.M., Vidal, A.M.: Parallel algorithms to compute the eigenvalues and eigenvectors of symmetric Toeplitz matrices. Parallel Algorithms Appl. 13, 75-93 (2000)

4. Bini, D., Di Benedetto, F.: Solving the generalized eigenvalue problem for rational Toeplitz matrices. SIAM J. Matrix Anal. Appl. 11, 537-552 (1990)

5. Bini, D., Pan, V.: Efficient algorithms for the evaluation of the eigenvalues of (block) banded Toeplitz matrices. Math. Comput. 50, 431-448 (1988)

6. Bogoya, J.M., Böttcher, A., Grudsky, S.M., Maximenko, E.A.: Eigenvalues of Hermitian Toeplitz matrices with smooth simple-loop symbols. J. Math. Anal. Appl. 422, 1308-1334 (2015)

7. Bogoya, J.M., Böttcher, A., Grudsky, S.M., Maximenko, E.A.: Maximum norm versions of the Szegő and Avram-Parter theorems for Toeplitz matrices. J. Approx. Theory 196, 79-100 (2015)

8. Bogoya, J.M., Grudsky, S.M., Maximenko, E.A.: Eigenvalues of Hermitian Toeplitz matrices generated by simple-loop symbols with relaxed smoothness. Oper. Theory Adv. Appl. 259, 179-212 (2017)

9. Böttcher, A., Silbermann, B.: Introduction to Large Truncated Toeplitz Matrices. Springer, New York (1999)

10. Böttcher, A., Grudsky, S.M., Maximenko, E.A.: Inside the eigenvalues of certain Hermitian Toeplitz band matrices. J. Comput. Appl. Math. 233, 2245-2264 (2010)

11. Brezinski, C., Redivo Zaglia, M.: Extrapolation Methods: Theory and Practice. North-Holland, Elsevier Science Publishers B.V., Amsterdam (1991)

12. Davis, P.J.: Interpolation and Approximation. Dover, New York (1975)

13. Ekström, S.-E., Garoni, C., Serra-Capizzano, S.: Are the eigenvalues of banded symmetric Toeplitz matrices known in almost closed form? Exper. Math. (in press). https://doi.org/10.1080/10586458. 2017.1320241

14. Garoni, C., Serra-Capizzano, S.: Generalized Locally Toeplitz Sequences: Theory and Applications, vol. I. Springer, Cham (2017)

15. Stoer, J., Bulirsch, R.: Introduction to Numerical Analysis, 3rd edn. Springer, New York (2010)

16. Trench, W.F.: On the eigenvalue problem for Toeplitz band matrices. Linear Algebra Appl. 64, 199214 (1985)

17. Trench, W.F.: Characteristic polynomials of symmetric rationally generated Toeplitz matrices. Linear Multilinear Algebra 21, 289-296 (1987)

18. Trench, W.F.: Numerical solution of the eigenvalue problem for symmetric rationally generated Toeplitz matrices. SIAM J. Matrix Anal. Appl. 9, 291-303 (1988)

19. Trench, W.F.: Numerical solution of the eigenvalue problem for Hermitian Toeplitz matrices. SIAM J. Matrix Anal. Appl. 10, 135-146 (1989)

20. Trench, W.F.: Numerical solution of the eigenvalue problem for efficiently structured Hermitian matrices. Linear Algebra Appl. 154-156, 415-432 (1991) 